\title{
Heat and Mass Transfer Impact on Differential Type Nanofluid with Carbon Nanotubes: A Study of Fractional Order System
}

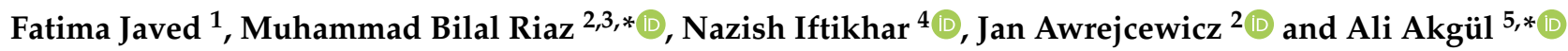 \\ 1 Department of Mathematics, University of Lahore, Lahore 54590, Pakistan; fatimajaved159@gmail.com \\ 2 Department of Automation, Biomechanics and Mechatronics, Lodz University of Technology, \\ 1/15 Stefanowskiego St., 90-924 Lodz, Poland; jan.awrejcewicz@p.lodz.pl \\ 3 Department of Mathematics, University of Management and Technology, Lahore 54770, Pakistan \\ 4 Department of Science \& Humanities, National University of Computer and Emerging Sciences, \\ Lahore 54770, Pakistan; nazish.iftikhar289@gmail.com \\ 5 Department of Mathematics, Art and Science Faculty, Siirt University, Siirt 56100, Turkey \\ * Correspondence: bilalsehole@gmail.com or muhammad.riaz@p.loz.pl (M.B.R.); aliakgul00727@gmail.com (A.A.)
}

\section{check for}

updates

Citation: Javed, F.; Riaz, M.B. Iftikhar, N.; Awrejcewicz, J.; Akgül, A. Heat and Mass Transfer Impact on Differential Type Nanofluid with Carbon Nanotubes: A Study of Fractional Order System. Fractal Fract 2021, 5, 231. https://doi.org/ $10.3390 /$ fractalfract5040231

Academic Editors: Carlo Cattani, Ming Li and Mohammad Hossein Heydari

Received: 12 October 2021

Accepted: 29 October 2021

Published: 18 November 2021

Publisher's Note: MDPI stays neutral with regard to jurisdictional claims in published maps and institutional affiliations.

Copyright: (c) 2020 by the authors. Licensee MDPI, Basel, Switzerland. This article is an open access article distributed under the terms and conditions of the Creative Commons Attribution (CC BY) license (https:// creativecommons.org/licenses/by/ $4.0 /$ )

\begin{abstract}
This paper is an analysis of flow of MHD CNTs of second grade nano-fluid under the influence of first order chemical reaction, suction, thermal generation and magnetic field. The fluid is flowing through a porous medium. For the study of heat and mass transfer, we applied the newly introduced differential operators to model such flow. The equations for heat, mass and momentum are established in the terms of Caputo (C), Caputo-Fabrizio (CF) and Atangana-Baleanu in Caputo sense $(A B C)$ fractional derivatives. This shows the novelty of this work. The equations for heat, mass and momentum are established in the terms of Caputo (C), Caputo-Fabrizio (CF) and AtanganaBaleanu in Caputo sense (ABC) fractional derivatives. The solutions are evaluated by employing Laplace transform and inversion algorithm. The flow in momentum profile due to variability in the values of parameters are graphically illustrated among $C, C F$ and $A B C$ models. It is concluded that fluid velocity showed decreasing behavior for $\chi, P, \hbar_{2}, M_{0}, P r, \aleph$ and $S C$ while it showed increasing behavior for $G r, G m, \mathcal{K}$ and $A_{0}$. Moreover, $A B C$ fractional operator presents larger memory effect than $C$ and $C F$ fractional operators.
\end{abstract}

Keywords: heat and mass transfer; power law; exponential law; nanofluid; carbon nanotube; nonlocal and non-singular kernel

\section{Introduction}

Nowadays, carbon nanotubes (CNTs) have fascinated researchers worldwide. Their stunning physical properties and very small dimensions makes them impressively useful in applied sciences, normal and artificial phenomena such as cooling instruments, crystal glowing, thermal exchange and various organic sciences. Palani and Abbas [1] observed MHD-free convection lambent flow over an oscillating plate. The convectional flow on a vertical conical sheet with impact of magnetic field was discussed by Kumar and Sivaraj [2] The impact of suction/injection on nano-fluid flow from two vertical sheets has been illustrated by Das et al. [3].

Through the past thirty years, fractional derivatives have fascinated multiple researchers as compared to classical derivatives. Furthermore, fractional derivatives are more credible in mathematical modeling of real world problems. The methodology of a fractional operator involves regular derivatives and kernel of fractional operator with convolution relation. Applications of fractional calculus have not been restricted to the disciplines of engineering and physical sciences, but also in other disciplines, such as ecology, geology, viscoelasticity, economics, probability and statistics, and fluid dynamics [4-7]. Cao et al. [8] analyzed a fractional model of nano-fluid over a moving plate. Pandey et al. [9] studied the effects of viscous dissipation and suction/injection on MHD flow of a nanofluid. At 
room temperature, thermal conductivity of $\mathrm{CNT}^{\prime}$ s nanofluid is six times greater than other material's nano fluid. This result has been discussed by Murshed et al. [10]. Abro et al. [11] applied a fractional derivative with non-singular and non-local kernel on a nano-fluid under magnetism. Saqib et al. [12] highlighted the strong memory effect of the AtanganaBaleanu fractional model of CNT's nano-fluid. The abeyance of nanoparticles in fluid airing notable enhancement of their properties at reticent nano-particle concentrations are known as nano-fluids [13]. Nanofluids parade enhanced thermal conductivity that escalates with growing volumetric fraction of nano-particles. In computers and other electronics, nanofluids are used to cool microchips. Lin et al. [14] inspected silver nanoparticles in vibrant thermal pipes. Saqib et al. [15] applied AB derivative to MHD flow of CMC based CNT's nano-fluid. Ikram et al. [16] diagnosed that Caputo fractional model exhibits a greater memory effect as compared to the Caputo-Fabrizio fractional model of nanofluid. Recently, Maiti et al. [17] studied the effect of the Caputo-Fabrizio derivative of fractional order model on the flow of blood in a porous tube having thermochemical properties under the magnetic and vibration mode.

The nanofluid with injection/suction, concentration and temperature are used in dissolution of garbage from nuclear reactors, filtration and absorption of chemicals by a soft medium. The motion of nanofluid under the impact of suction/injection in conical domain was discussed by Sreedevi et al. [18]. Du et al. [19] investigated the memory effect with derivative's order. Hayat et al. [20] inspected 3D flow of nanofluid under the influence of heat generation and magnetic field. CNTs have significant application in solar system. Ghalandari et al. [21] studied how nanofluids can be used in solar system. Nanofluids have many applications in stability, phase diagram, rheology and electroosmotic flow [22-24]. Alawi et al. [25] analyzed the thermophysical properties and stability of carbon nanostructures and metallic oxides nanofluids.

Pandikunta et al. [26] revealed that non-Newtonian MWCNTs Tangent hyperbolic nano-fluid minimizes the friction near the stretching sheet contrasting SWCNTs. The 4th order Runge-Kutta method was applied by Aghamajidi et al. [27] to MHD nano-flow model adjacent to a spinning down pointing vertical cone. Reddy et al. [28] analyzed MHD CNTs nanofluid flow over a stretching sheet and found that by increasing the value of thermal radiation parameter, the rate of heat transfer also increases. Upreti et al. [29] canvassed the flow and heat profile of 2-D CNTs nanofluid under the influence of magnetic field and blow / suction. Upreti et al. [30] designed a MHD model of 3D Darcy-Forchheimer flow of water-CNTs nanofluid and applied the Runge-Kutta-Fehlberg method to find the effects of Ohmic heating. Hussain et al. [31] considered single and multiple walled CNTs and cross-examined that thermal generation variable is inversely proportional to wall heat flux. Alsagri et al. [32] discovered that the dimensions of nano particles, thermal efficiency and volumetric fraction are directly proportional to each other in MHD finite film flow of human blood with CNTs nanofluids. Recently, the Atangana-Baleanu fractional model that is the finest fractional MHD rate type fluid model to reveal the memory effect of velocity and thermal distribution was discovered by Kumam et al. [33]. Saqib et al. [34] investigated flow of nanofluid under the influence of heat generation, magnetic field, shape effect of nanoparticles and thermal radiation. The velocity profile of Jeffery nanofluid was discussed by Roohi et al. [35] through a porous medium and under the influence of thermochemical effects. Fractional order model of thermo-solutal and magnetic nanoparticles transport for drug delivery applications has been investigated by authors [36]. Abro [37] explored a fractal-fractional derivative on ferromagnetic fluid via fractal Laplace transform.

This paper reveals the study of three different fractional models to show the memory effect of CNT's nanofluid model along with porosity, chemical reaction and suction/injection. The impact of emerging parameters for momentum, mass and energy solutions are plotted by different graphs with real justifications. Finally, a comparison has been made among C, $C F$ and $A B C$ fractional models. Fractional models of CNTs nanofluids have been rarely discussed due to their complexity. It has been proven in many already published articles that the heat and mass transfer do not really or always follow the classical mechanics 
process that is known as a memoryless process. Therefore, the model using classical differentiation based on the rate of change cannot really replicate such dynamical process very accurately; thus, a different concept of differentiation is needed to capture such processes. Very recently, new classes of differential operators were introduced and have been recognized to be efficient in capturing processes following the power law, the decay law and the crossover behaviors. We use these laws, which shows the novelty of our work.

\section{Mathematical Model}

Suppose a differential type nanofluid with carbon nanotube on a flat vertical plate under the influence of constant magnetic field $M_{o}$. Initially, the nanofluid and plate are at rest with ambient temperature $\widetilde{Y}_{\infty}$ and ambient concentration $\widetilde{\Lambda}_{\infty}$, respectively. As time increases from 0 , the plate starts vibrating with velocity $U_{o} d(\tau)$. As $\}$-axis is normal to plane, therefore the flow field depends only on time $\tau$ and $j$. The suction or injection along the direction of the fluid flow is constant, that is $v=-v_{0}$. The physical model of the problem can be given as follows [38] and its geometry is shown in Figure 1:

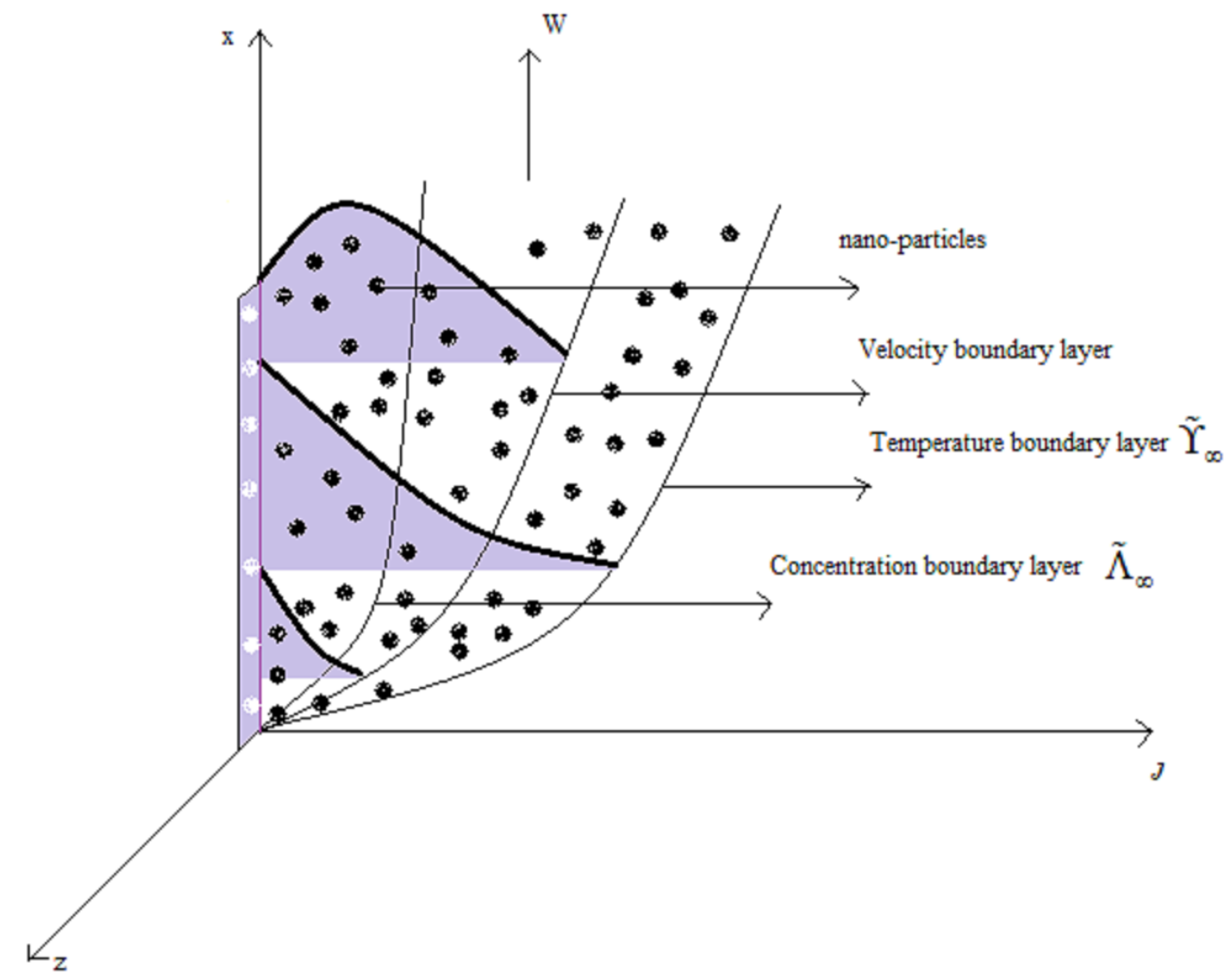

Figure 1. Physical model.

$$
\begin{aligned}
\rho_{n f} \partial_{\tau} \widetilde{W}(j, \tau)-\rho_{n f} v_{o} \partial_{j} \widetilde{W}(j, \tau)= & \mu_{n f} \partial_{j \jmath}^{2} \widetilde{W}(j, \tau)+\mu_{n f} \hbar \partial_{\tau \jmath j}^{3} \widetilde{W}(j, \tau)-\sigma M_{o} \widetilde{W}(j, \tau)-\frac{\mu_{n f} \wp}{P_{1}} \widetilde{W}(j, \tau) \\
& +g\left[\left(\rho \beta_{\widetilde{Y}}\right)_{n f}\left(\widetilde{Y}(j, \tau)-\widetilde{Y} \widetilde{Y}_{\infty}\right)+\left(\rho \beta_{\widetilde{\Lambda}}\right)_{n f}\left(\widetilde{\Lambda}(j, \tau)-\widetilde{\Lambda}_{\infty}\right)\right], \\
\partial_{\tau} \widetilde{Y}(j, \tau)-v_{o} \partial_{j} \widetilde{Y}(j, \tau)= & \left.\frac{k_{n f}}{\left(\rho C_{p}\right)_{n f}} \partial_{j \jmath}^{2} \widetilde{Y}(j, \tau)+\frac{A_{o}}{\left(\rho C_{p}\right)}(\widetilde{Y}(j, \tau)-\widetilde{Y})_{\infty}\right), \\
\partial_{\tau} \widetilde{\Lambda}(j, \tau)-v_{o} \partial_{j} \widetilde{\Lambda}(j, \tau)= & D \partial_{j \jmath}^{2} \widetilde{\Lambda}(j, \tau)+\kappa_{r}\left(\widetilde{\Lambda}(j, \tau)-\widetilde{\Lambda}_{\infty}\right) .
\end{aligned}
$$


The suitable initial and boundary conditions are

$$
\begin{aligned}
& \widetilde{W}(\jmath, 0)=0, \widetilde{Y}(\jmath, 0)=\widetilde{Y}_{\infty}, \widetilde{\Lambda}(\jmath, 0)=\widetilde{\Lambda}_{\infty}, \\
& \widetilde{W}(0, \tau)=U_{o} d(\tau), \widetilde{Y}(0, \tau)=\widetilde{Y}_{w}, \widetilde{\Lambda}(0, \tau)=\widetilde{\Lambda}_{w}, \\
& \widetilde{W}(\jmath, \tau)=0, \widetilde{Y}(\jmath, \tau)=\widetilde{Y}_{\infty}, \widetilde{\Lambda}(\jmath, \tau)=\widetilde{\Lambda}_{\infty}, \text { as } \jmath \rightarrow \infty .
\end{aligned}
$$

Dimensionless variables are given below

$$
\begin{gathered}
j^{*}=\frac{U_{o f}}{v_{n f}}, \tau^{*}=\frac{U_{o}^{2} \tau}{v_{n f}}, W^{*}=\frac{\widetilde{W}}{U_{o}}, Y^{*}=\frac{\widetilde{Y}-\widetilde{Y}_{\infty}}{\widetilde{Y}_{w}-\widetilde{Y}_{\infty}}, \Lambda^{*}=\frac{\widetilde{\Lambda}-\widetilde{\Lambda}_{\infty}}{\widetilde{\Lambda}_{w}-\widetilde{\Lambda}_{\infty}}, \operatorname{Pr}=\frac{\mu C_{p}}{k}, \\
S c=\frac{v_{n f}}{D_{m}}, G r=\frac{g\left(v \beta_{\widetilde{Y}}\right)_{n f}\left(\widetilde{Y}_{w}-\widetilde{Y}_{\infty}\right)}{U_{o}^{3}}, G m=\frac{g\left(v \beta_{\widetilde{\Lambda}}\right)_{n f}\left(\widetilde{\Lambda}_{w}-\widetilde{\Lambda}_{\infty}\right)}{U_{o}^{3}}, \\
M_{o}^{*}=\frac{\sigma M_{o} v_{n f}}{U_{o}^{2}}, P=\frac{\left(v_{n f}\right)^{2} \wp}{P_{1} U_{o}^{2}}, A_{o}^{*}=\frac{A_{o} v_{n f}}{\rho C_{p} U_{o}^{2}}, \kappa=\frac{\kappa_{r} v_{n f}}{U_{o}^{2}}, \aleph=\frac{v_{o}}{U_{o}},
\end{gathered}
$$

and dimensionless set of governing equations are (ignoring *):

$$
\begin{aligned}
\partial_{\tau} W(\jmath, \tau)-\aleph \partial_{j} W(\jmath, \tau)= & \partial_{\jmath \jmath}^{2} W(\jmath, \tau)+\hbar_{2} \partial_{\tau \jmath \jmath}^{3} W(\jmath, \tau)+\operatorname{Gr} Y(\jmath, \tau) \\
& +G m \Lambda(\jmath, \tau)-M_{o} W(\jmath, \tau)-\frac{1}{P} W(\jmath, \tau), \\
\partial_{\tau} Y(\jmath, \tau)-\aleph \partial_{\jmath} Y(\jmath, \tau)= & \frac{1}{P r} \partial_{\jmath \jmath}^{2} Y(\jmath, \tau)+A_{o} Y(\jmath, \tau), \\
\partial_{\tau} \Lambda(\jmath, \tau)-\aleph \partial_{\jmath} \Lambda(\jmath, \tau)= & \frac{1}{S c} \partial_{j \jmath}^{2} \Lambda(\jmath, \tau)+\kappa \Lambda(\jmath, \tau) .
\end{aligned}
$$

Furthermore, dimensionless initial and boundary conditions are:

$$
\begin{aligned}
& W(\jmath, 0)=0, Y(\jmath, 0)=0, \Lambda(\jmath, 0)=0, \\
& W(0, \tau)=d(\tau), Y(0, \tau)=1, \Lambda(0, \tau)=1, \\
& W(\jmath, \tau)=0, Y(\jmath, \tau)=0, \Lambda(\jmath, \tau)=0, \text { as } \jmath \rightarrow \infty .
\end{aligned}
$$

Preliminaries

For solutions for fractional order models, we will use the definitions given below: The Caputo time derivative is defined as [39]:

$$
{ }^{C} D_{\tau}^{\chi} m(\jmath, \tau)=\frac{1}{\Xi(i-\chi)} \int_{b}^{\tau}\left(\frac{g^{(i)}(\varrho)}{(\tau-\varrho)^{\chi+1-i}}\right) d \varrho,
$$

where $\Xi(\cdot)$ is gamma function.

By applying the Laplace transform to Caputo $(C)$ derivative, we get

$$
\mathcal{L}\left({ }^{C} D_{\tau}^{\chi} m(\jmath, \tau)\right)=u^{\chi} \mathcal{L}(m(\jmath, \tau))-u^{\chi-1} g(\jmath, 0) .
$$

The Caputo-Fabrizio (CF) time derivative is defined as [40]:

$$
{ }^{C F} D_{\tau}^{\chi} m(\jmath, \tau)=\frac{G(\chi)}{1-\chi} \int_{b}^{\tau} \exp \left(-\frac{\chi(\tau-\chi)}{1-\chi}\right) \frac{\partial g(\jmath, \varrho)}{\partial \varrho} d \varrho,
$$

where $G(\chi)$ is a normalization function/constant depending on b and here we consider $G(\chi)=1$. 
Applying the Laplace transform to $C F$ derivative, we get

$$
\mathcal{L}\left({ }^{C F} D_{\tau}^{\chi} m(\jmath, \tau)\right)=\frac{u \mathcal{L}(m(\jmath, \tau))-g(\jmath, 0)}{(1-\chi) u+\chi} .
$$

The Atangana-Baleanu $(A B C)$ time derivative in Caputo sense is defined as [41,42]:

$$
{ }^{A B C} D_{\tau}^{\chi} m(\jmath, \tau)=\frac{A B(\chi)}{1-\chi} \int_{b}^{\tau} E_{\chi}\left(-\frac{\chi(\tau-\chi)}{1-\chi}\right) \frac{\partial g(\jmath, \varrho)}{\partial \varrho} d \varrho,
$$

where $A B(\chi)$ is a normalization function/constant depending on a and here we consider $A B(\chi)=1$.

The Laplace transform of $A B C$ derivative is

$$
\mathcal{L}\left({ }^{A B C} D_{\tau}^{\chi} m(\jmath, \tau)\right)=\frac{u^{\chi} \mathcal{L}(m(\jmath, \tau))-u^{\chi-1} g(\jmath, 0)}{(1-\chi) u^{\chi}+\chi} .
$$

\section{Solutions via Caputo}

Following are the fractional solutions of heat, mass and flow with Caputo fractional derivative.

\subsection{Temperature Profile}

By using definition of Caputo derivative Equation (10), we have

$$
{ }^{C} D_{\tau} \mathrm{Y}(\jmath, \tau)-\aleph \partial_{\jmath} \mathrm{Y}(\jmath, \tau)=\frac{1}{\operatorname{Pr}} \partial_{\jmath \jmath}^{2} \mathrm{Y}(\jmath, \tau)+A_{o} \mathrm{Y}(\jmath, \tau) .
$$

Taking Laplace of Equation (16) by using Equation (11)

$$
u^{\chi} \overline{\mathrm{Y}}_{C}(\jmath, u)-\aleph \partial_{\jmath} \overline{\mathrm{Y}}_{C}(\jmath, u)=\frac{1}{\operatorname{Pr}} \partial_{\jmath \jmath}^{2} \overline{\mathrm{Y}}_{C}(\jmath, u)+A_{o} \overline{\mathrm{Y}}_{C}(\jmath, u) .
$$

Solution of Equation (17) is

$$
\bar{Y}_{C}(\jmath, u)=\frac{1}{u} e^{-\left[\frac{\aleph P r}{2}+\sqrt{\frac{(\aleph P r)^{2}+P r\left(u \chi-A_{0}\right)}{4}}\right] .}
$$

\subsection{Concentration Profile}

Concentration field with $C$ time-fractional derivative is given by applying Equation (10), we have

$$
{ }^{C} D_{\tau} \Lambda(\jmath, \tau)-\aleph \partial_{j} \Lambda(\jmath, \tau)=\frac{1}{S c} \partial_{j \jmath}^{2} \Lambda(\jmath, \tau)+\kappa \Lambda(\jmath, \tau) .
$$

Taking Laplace of Equation (19) by using Equation (11), we get

$$
u^{\chi} \bar{\Lambda}_{C}(\jmath, u)-\aleph \partial_{j} \bar{\Lambda}_{C}(\jmath, u)=\frac{1}{S c} \partial_{j \jmath}^{2} \bar{\Lambda}_{C}(\jmath, u)+\kappa \bar{\Lambda}_{C}(\jmath, u) .
$$

Solution of Equation (20) is

$$
\bar{\Lambda}_{C}(\jmath, u)=\frac{1}{u} e^{-\jmath\left[\frac{\aleph S c}{2}+\sqrt{\frac{(\aleph S c)^{2}+S c(u X-\kappa)}{4}}\right] .}
$$




\subsection{Velocity Profile}

Velocity field with $C$ time-fractional derivative is given by applying Equation (10), we have

$$
\begin{array}{r}
{ }^{C} D_{\tau} W(\jmath, \tau)-\aleph \partial_{\jmath} W(\jmath, \tau)=\partial_{\jmath \jmath}^{2} W(\jmath, \tau)+\hbar_{2}{ }^{C} D_{\tau} \partial_{j \jmath}^{2} W(\jmath, \tau)+G r Y(\jmath, \tau) \\
+G m \Lambda(\jmath, \tau)-M_{o} W(\jmath, \tau)-\frac{1}{P} W(\jmath, \tau) .
\end{array}
$$

Taking Laplace of Equation (22) by using Equation (11), we get

$$
\begin{array}{r}
u^{\chi} \bar{W}_{C}(\jmath, u)-\aleph \partial_{j} \bar{W}_{C}(\jmath, u)=\partial_{\jmath \jmath}^{2} \bar{W}_{C}(\jmath, u)+\hbar_{2} u^{\chi} \partial_{\jmath \jmath}^{2} \bar{W}_{C}(\jmath, u)+G r \bar{Y}_{C}(\jmath, u)+G m \bar{\Lambda}_{C}(\jmath, u) \\
-M_{o} \bar{W}_{C}(\jmath, u)-\frac{1}{P} \bar{W}_{C}(\jmath, u),
\end{array}
$$

Solution of Equation (23) is

$$
\begin{aligned}
\bar{W}_{C}(\jmath, u)=d\left(u^{\chi}\right) e^{-\jmath F_{1}} & +G r\left[\frac{e^{-\jmath F_{1}}-e^{-\jmath A_{1}}}{u\left[\left(1+\hbar_{2} u \chi\right) A_{1}^{2}+\aleph A_{1}-\left(M_{0}+\frac{1}{P}+u \chi\right)\right]}\right] \\
& +G m\left[\frac{e^{-\jmath F_{1}}-e^{-\jmath B_{1}}}{u\left[\left(1+\hbar_{2} u \chi\right) B_{1}^{2}+\aleph B_{1}-\left(M_{o}+\frac{1}{P}+u \chi\right)\right]}\right],
\end{aligned}
$$

where

$$
\begin{gathered}
F_{1}\left(u^{\chi}\right)=\frac{1}{2}\left[\frac{\aleph}{1+\hbar_{2} u^{\chi}}+\sqrt{\left(\frac{\aleph}{1+\hbar_{2} u^{\chi}}\right)^{2}+\frac{4}{1+\hbar_{2} u^{\chi}}\left(M_{o}+\frac{1}{P}+u^{\chi}\right)}\right], \\
A_{1}\left(u^{\chi}\right)=\frac{1}{2}\left[\aleph P r+\sqrt{(\aleph P r)^{2}+4 \operatorname{Pr}\left(u^{\chi}-A_{o}\right)}\right] \\
B_{1}\left(u^{\chi}\right)=\frac{1}{2}\left[\aleph S c+\sqrt{(\aleph S c)^{2}+4 S c\left(u^{\chi}-\kappa\right)}\right] .
\end{gathered}
$$

\section{Solutions via Caputo-Fabrizio}

The non-integer order solutions via singular kernel Caputo-Fabrizio fractional derivative for temperature, concentration and velocity are as follow:

\subsection{Temperature Profile}

Applying definition of Caputo-Fabrizio (12) to non-dimensional heat equation

$$
{ }^{C F} D_{\tau} Y(j, \tau)-\aleph \partial_{j} Y(\jmath, \tau)=\frac{1}{P r} \partial_{j \jmath}^{2} Y(j, \tau)+A_{o} Y(j, \tau),
$$

and applying Laplace Equation (13) to above Equation (28)

$$
\frac{u}{(1-\chi) u+\chi} \overline{\mathrm{Y}}_{C F}(\jmath, u)-\aleph \partial_{j} \overline{\mathrm{Y}}_{C F}(\jmath, u)=\frac{1}{P r} \partial_{\jmath \jmath}^{2} \overline{\mathrm{Y}}_{C F}(\jmath, u)+A_{o} \overline{\mathrm{Y}}_{C F}(\jmath, u),
$$

Solution of Equation (29) is

$$
\bar{Y}_{C F}(\jmath, u)=\frac{1}{u} e^{-\jmath\left[\frac{\aleph P r}{2}+\sqrt{\frac{(\aleph P r)^{2}+\operatorname{Pr}\left(\frac{u}{(1-\chi) u+\chi}-A_{0}\right)}{4}}\right]} .
$$




\subsection{Concentration Profile}

Applying definition of Caputo-Fabrizio (12) to non-dimensional mass equation

$$
{ }^{C F} D_{\tau} \Lambda(\jmath, \tau)-\aleph \partial_{j} \Lambda(\jmath, \tau)=\frac{1}{S c} \partial_{\jmath \jmath}^{2} \Lambda(\jmath, \tau)+\kappa \Lambda(\jmath, \tau),
$$

and applying Laplace Equation (13) to above Equation (31)

$$
\frac{u}{(1-\chi) u+\chi} \bar{\Lambda}_{C F}(j, u)-\aleph \partial_{\jmath} \bar{\Lambda}_{C F}(j, u)=\frac{1}{S c} \partial_{j \jmath}^{2} \bar{\Lambda}_{C F}(\jmath, u)+\kappa \bar{\Lambda}_{C F}(\jmath, u),
$$

The solution of Equation (32) is

$$
\bar{\Lambda}_{C F}(\jmath, u)=\frac{1}{u} e^{-\jmath\left[\frac{\aleph S c}{2}+\sqrt{\frac{(\aleph S c)^{2}+S c\left(\frac{u}{(1-\chi) u+\chi}-\kappa\right)}{4}}\right]} .
$$

\subsection{Velocity Profile}

Applying definition of Caputo-Fabrizio (12) to non-dimensional flow equation

$$
\begin{array}{r}
{ }^{C F} D_{\tau} W(\jmath, \tau)-\aleph \partial_{j} W(\jmath, \tau)=\partial_{\jmath \jmath}^{2} W(\jmath, \tau)+\hbar_{2}{ }^{C F} D_{\tau} \partial_{\jmath \jmath}^{2} W(\jmath, \tau)+G r Y(\jmath, \tau) \\
+G m \Lambda(\jmath, \tau)-M_{o} W(\jmath, \tau)-\frac{1}{P} W(\jmath, \tau) .
\end{array}
$$

Taking Laplace of Equation (34), we get

$$
\begin{aligned}
\frac{u}{(1-\chi) u+\chi} \bar{W}_{C F}(j, u)-\aleph \partial_{j} \bar{W}_{C F}(\jmath, u)= & \partial_{\jmath \jmath}^{2} \bar{W}_{C F}(\jmath, u)+\hbar_{2} \frac{u}{(1-\chi) u+\chi} \partial_{\jmath \jmath}^{2} \bar{W}_{C F}(\jmath, u) \\
& +G r \bar{Y}_{C F}(\jmath, u)+G m \overline{\Lambda_{C F}}(j, u)-M_{o} \bar{W}_{C F}(j, u) \\
& -\frac{1}{P} \bar{W}_{C F}(\jmath, u),
\end{aligned}
$$

The solution of Equation (35) is

$$
\begin{aligned}
\bar{W}_{C F}(\jmath, u)= & d\left(u^{\chi}\right) e^{-\jmath F_{2}}+G r\left[\frac{e^{-\jmath F_{2}}-e^{-\jmath A_{2}}}{u\left[\frac{b u+\chi}{(1-\chi) u+\chi} A_{2}^{2}+\aleph A_{2}-\left(M_{o}+\frac{1}{P}+\frac{u}{(1-\chi) u+\chi}\right)\right]}\right] \\
& +G m\left[\frac{e^{-\jmath F_{2}}-e^{-\jmath B_{2}}}{u\left[\frac{b u+\chi}{(1-\chi) u+\chi} B_{2}^{2}+\aleph B_{2}-\left(M_{o}+\frac{1}{P}+u^{\chi} \frac{u}{(1-\chi) u+\chi}\right)\right]}\right],
\end{aligned}
$$

where

$$
\begin{gathered}
F_{2}\left(u^{\chi}\right)=\frac{1}{2}\left[\aleph \frac{(1-\chi) u+\chi}{b u+\chi}+\sqrt{\left(\aleph \frac{(1-\chi) u+\chi}{b u+\chi}\right)^{2}+4 \aleph \frac{(1-\chi) u+\chi}{b u+\chi}\left(M_{o}+\frac{1}{P}+\frac{u}{(1-\chi) u+\chi}\right)}\right], \\
A_{2}\left(u^{\chi}\right)=\frac{1}{2}\left[\aleph \operatorname{Pr}+\sqrt{(\aleph P r)^{2}+4 \operatorname{Pr}\left(\frac{u}{(1-\chi) u+\chi}-A_{o}\right)}\right], \\
B_{2}\left(u^{\chi}\right)=\frac{1}{2}\left[\aleph S c+\sqrt{(\aleph S c)^{2}+4 S c\left(\frac{u}{(1-\chi) u+\chi}-\kappa\right)}\right] .
\end{gathered}
$$




\section{Solutions via Atangana Baleanu}

The non-integer order solutions via Atangana-Baleanu derivative of temperature, concentration and velocity are as follow:

\subsection{Temperature Profile}

Applying definition of Atangana-Baleanu (14) to non-dimensional heat equation

$$
{ }^{A B C} D_{\tau} \mathrm{Y}(\jmath, \tau)-\aleph \partial_{\jmath} \mathrm{Y}(\jmath, \tau)=\frac{1}{\operatorname{Pr}} \partial_{\jmath \jmath}^{2} \mathrm{Y}(\jmath, \tau)+A_{o} \mathrm{Y}(\jmath, \tau),
$$

and applying Laplace (15) to above Equation (40)

$$
\frac{u^{\chi}}{(1-\chi) u^{\chi}+\chi} \overline{\mathrm{Y}}_{A B C}(\jmath, u)-\aleph \partial_{\jmath} \overline{\mathrm{Y}}_{A B C}(\jmath, u)=\frac{1}{\operatorname{Pr}} \partial_{\jmath \jmath}^{2} \overline{\mathrm{Y}}_{A B C}(\jmath, u)+A_{o} \overline{\mathrm{Y}}_{A B C}(\jmath, u),
$$

Solution of Equation (41) is

$$
\overline{\mathrm{Y}}_{A B C}(\jmath, u)=\frac{1}{u} e^{-\jmath\left[\frac{\aleph P r}{2}+\sqrt{\left.\frac{(\aleph P r)^{2}+\operatorname{Pr}\left(\frac{u \chi}{(1-\chi) u X}-\bar{x}\right.}{4}-A_{0}\right)}\right]} .
$$

\subsection{Concentration Profile}

Applying definition of Atangana-Baleanu (14) to non-dimensional mass equation

$$
{ }^{A B C} D_{\tau} \Lambda(\jmath, \tau)-\aleph \partial_{\jmath} \Lambda(\jmath, \tau)=\frac{1}{S c} \partial_{\jmath \jmath}^{2} \Lambda(\jmath, \tau)+\kappa \Lambda(\jmath, \tau),
$$

and applying Laplace (15) to above Equation (43)

$$
\frac{u^{\chi}}{(1-\chi) u \chi+\chi} \bar{\Lambda}_{A B C}(\jmath, u)-\aleph \partial_{\jmath} \bar{\Lambda}_{A B C}(\jmath, u)=\frac{1}{S c} \partial_{\jmath \jmath}^{2} \bar{\Lambda}_{A B C}(\jmath, u)+\kappa \bar{\Lambda}_{A B C}(\jmath, u),
$$

The solution of Equation (44) is

$$
\bar{\Lambda}_{A B C}(\jmath, u)=\frac{1}{u} e^{-\jmath\left[\frac{\aleph S c}{2}+\sqrt{\frac{(\aleph S c)^{2}+S c\left(\frac{u \chi}{(1-\chi) u X+\chi}-\kappa\right)}{4}}\right] .} .
$$

\subsection{Velocity Profile}

Applying definition of Atangana-Baleanu (14) to non-dimensional flow equation

$$
\begin{aligned}
{ }^{A B C} D_{\tau} W(\jmath, \tau)-\aleph \partial_{\jmath} W(\jmath, \tau)= & \partial_{\jmath \jmath}^{2} W(\jmath, \tau)+\hbar_{2}{ }^{A B C} D_{\tau} \partial_{\jmath \jmath}^{2} W(\jmath, \tau)+G r Y(\jmath, \tau) \\
& +G m \Lambda(\jmath, \tau)-M_{o} W(\jmath, \tau)-\frac{1}{P} W(\jmath, \tau) .
\end{aligned}
$$

Taking Laplace of Equation (46), we get

$$
\begin{aligned}
\frac{u^{\chi}}{(1-\chi) u \chi+\chi} \bar{W}_{A B C}(\jmath, u)-\aleph \partial_{j} \bar{W}_{A B C}(\jmath, u)= & \partial_{j \jmath}^{2} \bar{W}_{A B C}(\jmath, u)+\hbar_{2} \frac{u^{\chi}}{(1-\chi) u \chi+\chi} \partial_{j \jmath}^{2} \bar{W}_{A B C}(\jmath, u) \\
& +G r \bar{Y}_{A B C}(\jmath, u)+G m \bar{\Lambda}_{A B C}(\jmath, u)-M_{o} \bar{W}_{A B C}(\jmath, u) \\
& -\frac{1}{P} \bar{W}_{A B C}(\jmath, u),
\end{aligned}
$$


Solution of Equation (47) is

$$
\begin{aligned}
\bar{W}_{A B C}(\jmath, u)= & d\left(u^{\chi}\right) e^{-\jmath F_{3}}+G r\left[\frac{e^{-\jmath F_{3}}-e^{-\jmath A_{3}}}{u\left[\frac{b u \chi+\chi}{(1-\chi) u^{\chi}+\chi} A_{3}^{2}+\aleph A_{3}-\left(M_{o}+\frac{1}{P}+\frac{u \chi}{(1-\chi) u^{\chi}+\chi}\right)\right]}\right] \\
& +G m\left[\frac{e^{-\jmath F_{3}}-e^{-\jmath B_{3}}}{u\left[\frac{b u \chi+\chi}{(1-\chi) u^{\chi}+\chi} B_{3}^{2}+\aleph B_{3}-\left(M_{o}+\frac{1}{P}+\frac{u^{\chi}}{(1-\chi) u^{\chi}+\chi}\right)\right]}\right]
\end{aligned}
$$

where

$$
F_{3}\left(u^{\chi}\right)=\frac{1}{2}\left[\aleph \frac{(1-\chi) u^{\chi}+\chi}{b u \chi+\chi}+\sqrt{\left(\aleph \frac{(1-\chi) u^{\chi}+\chi}{b u \chi}\right)^{2}+4 \aleph \frac{(1-\chi) u^{\chi}+\chi}{b u^{\chi}+\chi}\left(M_{o}+\frac{1}{P}+\frac{u^{\chi}}{(1-\chi) u^{\chi}+\chi}\right)}\right]
$$

$$
\begin{aligned}
& A_{3}\left(u^{\chi}\right)=\frac{1}{2}\left[\aleph \operatorname{Pr}+\sqrt{(\aleph \operatorname{Pr})^{2}+4 \operatorname{Pr}\left(\frac{u^{\chi}}{(1-\chi) u^{\chi}+\chi}-A_{o}\right)}\right] \\
& B_{3}\left(u^{\chi}\right)=\frac{1}{2}\left[\aleph S c+\sqrt{(\aleph S c)^{2}+4 S c\left(\frac{u^{\chi}}{(1-\chi) u^{\chi}+\chi}-\kappa\right)}\right]
\end{aligned}
$$

The results obtained for velocity, temperature and concentration after solving the fractionalized models are complex and generalized in nature. As the models were fractionalized with power Law, exponential and non-local kernel to see the significance of memory effects. These are generalized results, and as the fractional parameter goes to 1 , we recovered the result calculated for integer order case. The detail is as by taking $\chi \rightarrow 1$ in Equations (18), (30) and (42), we will get similar results as for integer order obtained by Ahmad et al. [38] [Equation (12)]. As $\chi$ approaches 1 in Equations (21), (33) and (45), identical results exist in Ahmad et al. [38] [Equation (17)]. Furthermore, as $\chi \rightarrow 1$ in Equations (24), (36) and (48), results for fractional order reduce to integer order presented by Ahmad et al. [38] [Equation (22)]. This shows worth of our results. As we mentioned early the roots are complex in nature in order to find their inverses we use inversion algorithm namely Stehfest's formula [43], one of the simplest algorithm we use to sort out the inverse Laplace transform.

$$
v(r, t)=\frac{e^{4.7}}{t}\left[\frac{1}{2} \bar{v}\left(r, \frac{4.7}{t}\right)+\operatorname{Re}\left\{\sum_{k=1}^{N_{1}}(-1)^{k} \bar{v}\left(r, \frac{4.7+k \pi i}{t}\right)\right\}\right],
$$

where $\operatorname{Re}($.$) is the real part, i$ is the imaginary unit and $N_{1}$ is a natural number.

\section{Results and Discussion}

MHD CNT's flow of nanofluid has been studied, in this paper for three particular time derivative $(C, C F$ and $A B C$ ). Fractional models of flow, heat and mass are introduced. Graphs has been drawn to ellaborate the effects of $\chi, G r, G m, \aleph, P r, S c, P, M_{0}, \hbar_{2}, A_{o}$ and $\kappa$ for fractional models.

\subsection{Effect of $\chi$}

From Figure 2, we can observe that velocity of nanofluid decreases as fractional parameter increases. The fractional models $C, C F$, and $A B C$ reduce to integer order model as $\chi$ tends to 1 . It is worth mentioning that fractional order models are best to explain the 
history (memory) of the fluids. As there is non-local and non-singular kernel present in Atangana-Baleanu time derivative, velocity is highest for $A B C$.
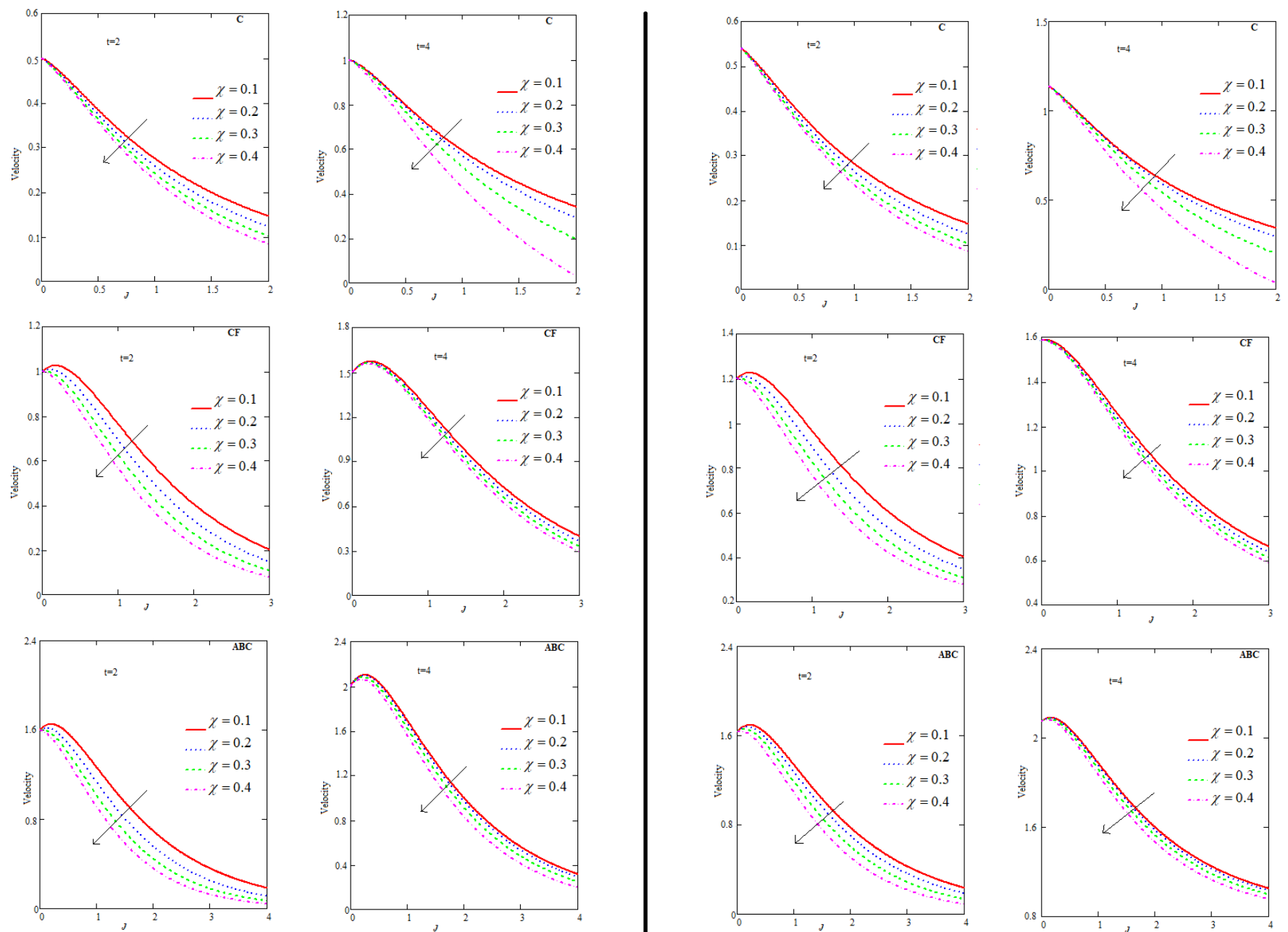

(a) Graphs for $d(t)=1$

(b) Graphs for $d(t)=e^{t}$

Figure 2. Graphs for two different cases of velocity with variable fractional parameter and $M_{o}=2, S c=1.2, \operatorname{Pr}=1$, $G m=5, G r=5, A_{0}=0.5, \hbar_{2}=0.7, \aleph=0.2, P=0.3$ and $\kappa=0.9$.

\subsection{Effect of $\mathrm{Gm}$}

From Figure 3, velocity of nano-fluid in CNT escalates as Gm increases. Apparently, augmenting buoyancy forces leads to decrease viscous forces and this causes reduction in frictional forces. Due to this physical phenomena, velocity increase. It is lowest for Caputo. 

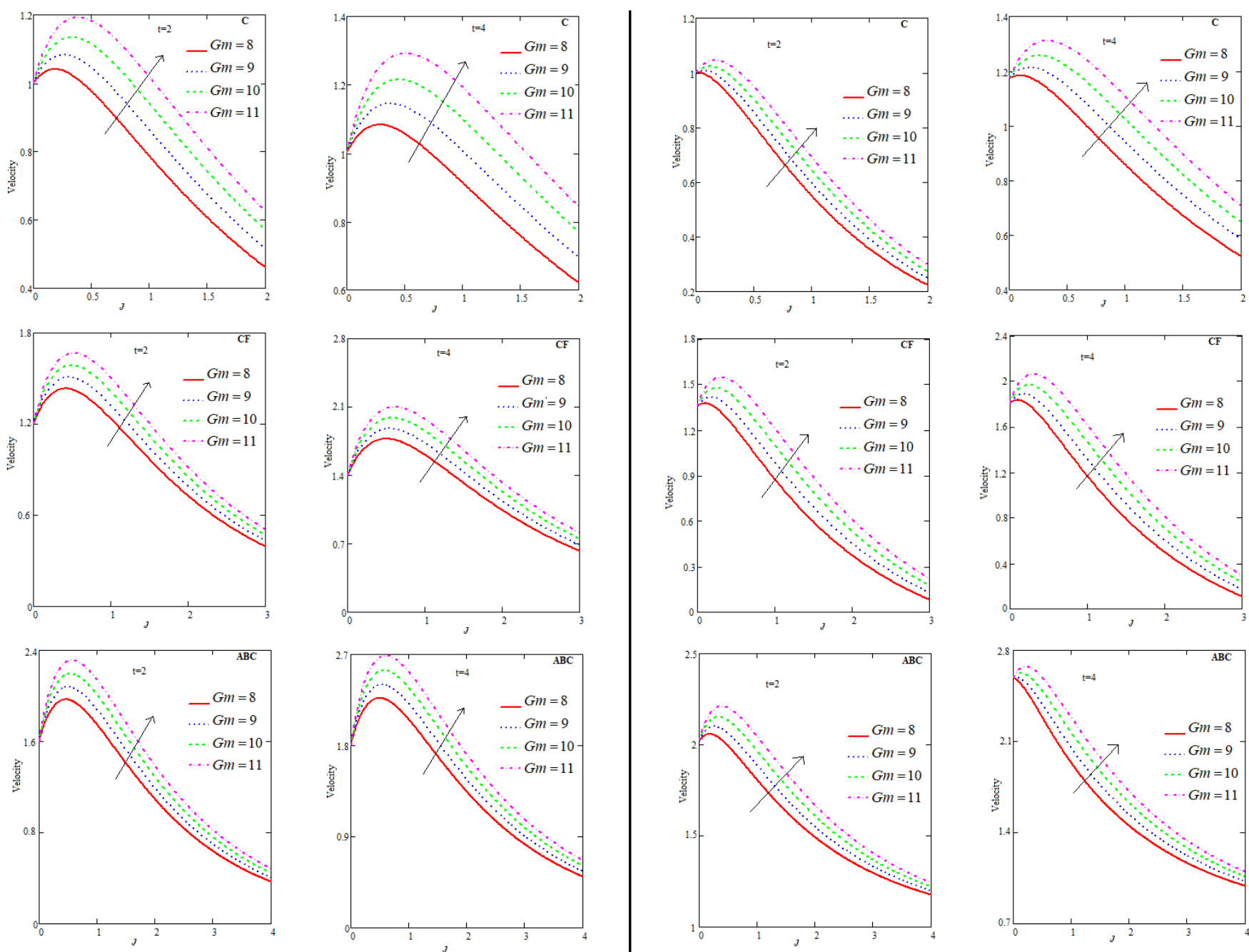

(a) Graphs for $d(t)=1$

(b) Graphs for $d(t)=e^{t}$

Figure 3. Graphs for two different cases of velocity with variable mass Grashof number and $M_{0}=2, S c=1.2, \operatorname{Pr}=1$, $\chi=0.1, G r=5, A_{o}=0.5, \hbar_{2}=0.7, \aleph=0.2, P=0.3$ and $\kappa=0.9$.

\subsection{Effect of $\mathrm{Gr}$}

From Figure 4, one can see that as $G r$ increases, the flow of nano-fluid increases.

Density of fluid decreases when buoyancy forces increases, so there is no or very less viscous forces which yields the increase in velocity profile. Among all three fractional derivatives, $A B C$ shows maximum velocity. 

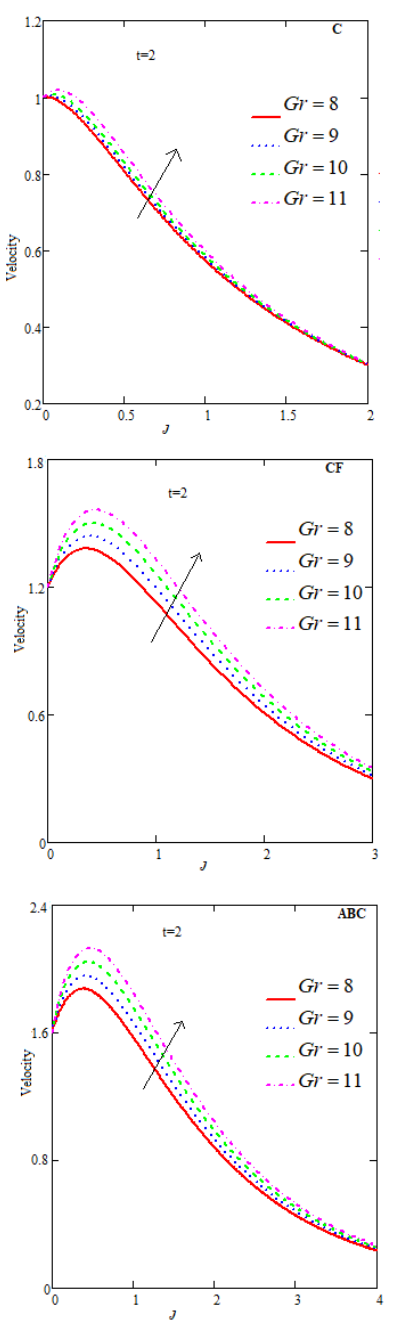

(a) Graphs for $d(t)=1$
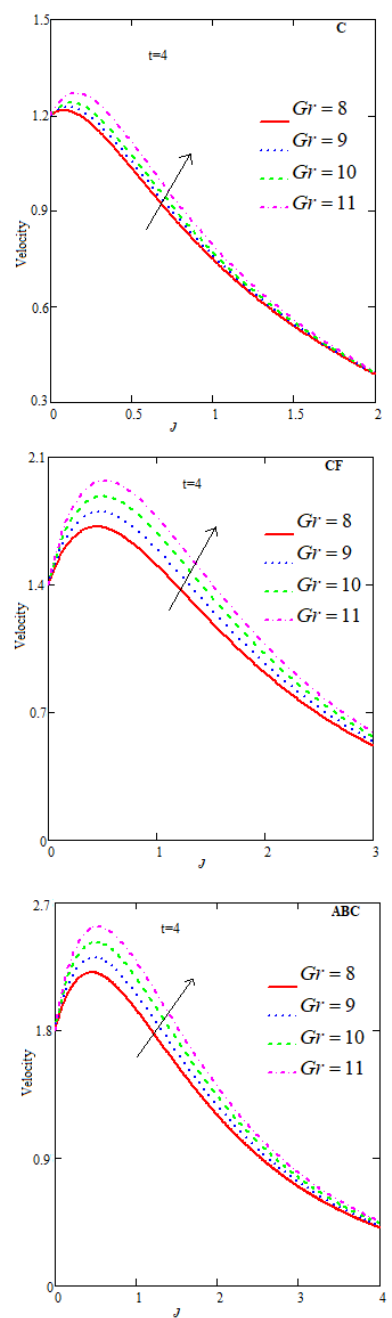
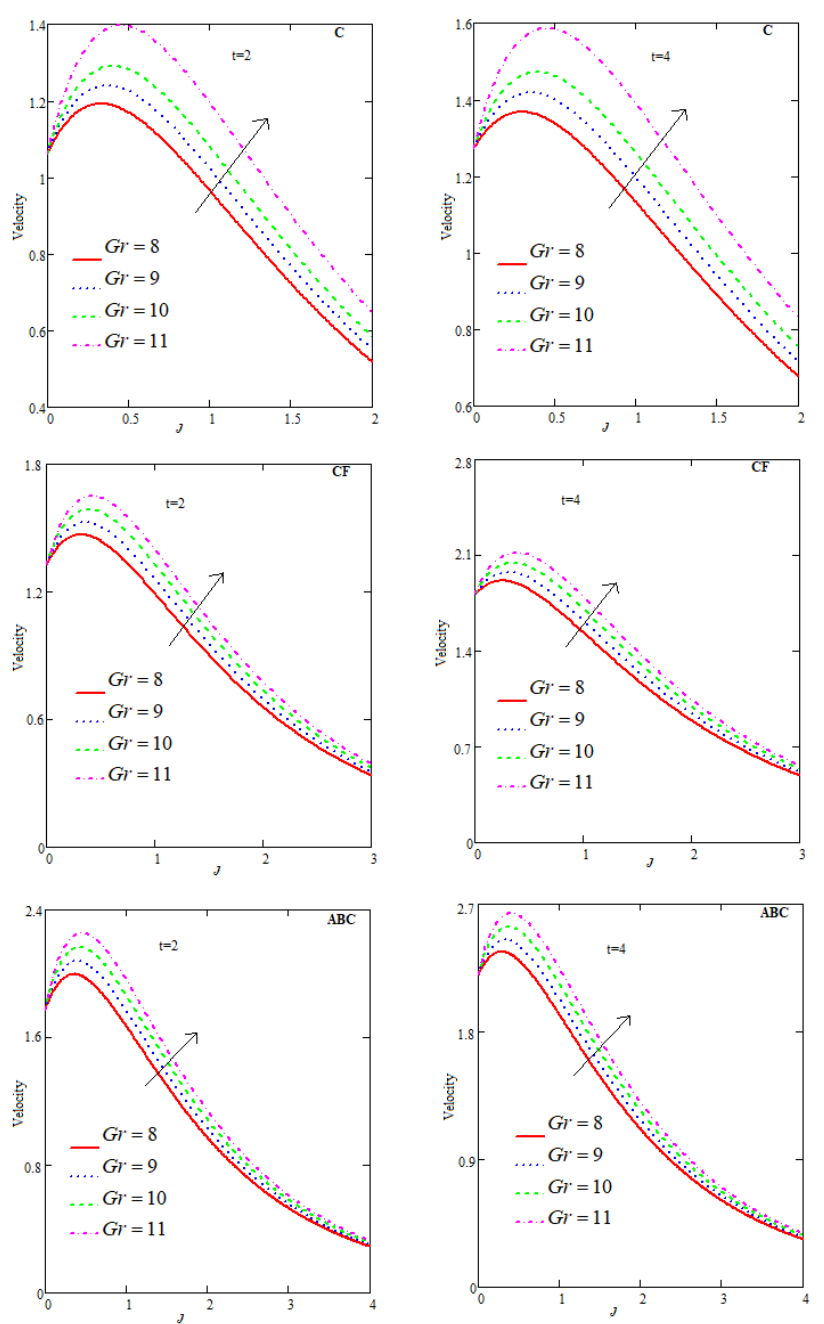

(b) Graphs for $d(t)=e^{t}$

Figure 4. Graphs for two different cases of velocity with variable thermal Grashof number and $M_{0}=2, S c=1.2, \operatorname{Pr}=1$, $\chi=0.1, \mathrm{Gm}=5, A_{o}=0.5, \hbar_{2}=0.7, \aleph=0.2, P=0.3$ and $\kappa=0.9$.

\subsection{Effect of $P$}

Figure 5 shows that as permittivity of medium increases, flow profile decreases. Apparently, $P$ is directly proportional to porosity. Hence, when medium becomes more porous, flow will reduce its speed. Among all three fractional derivatives, $A B C$ fractional model shows highest velocity as permittivity increases simultaneously as time varies. 

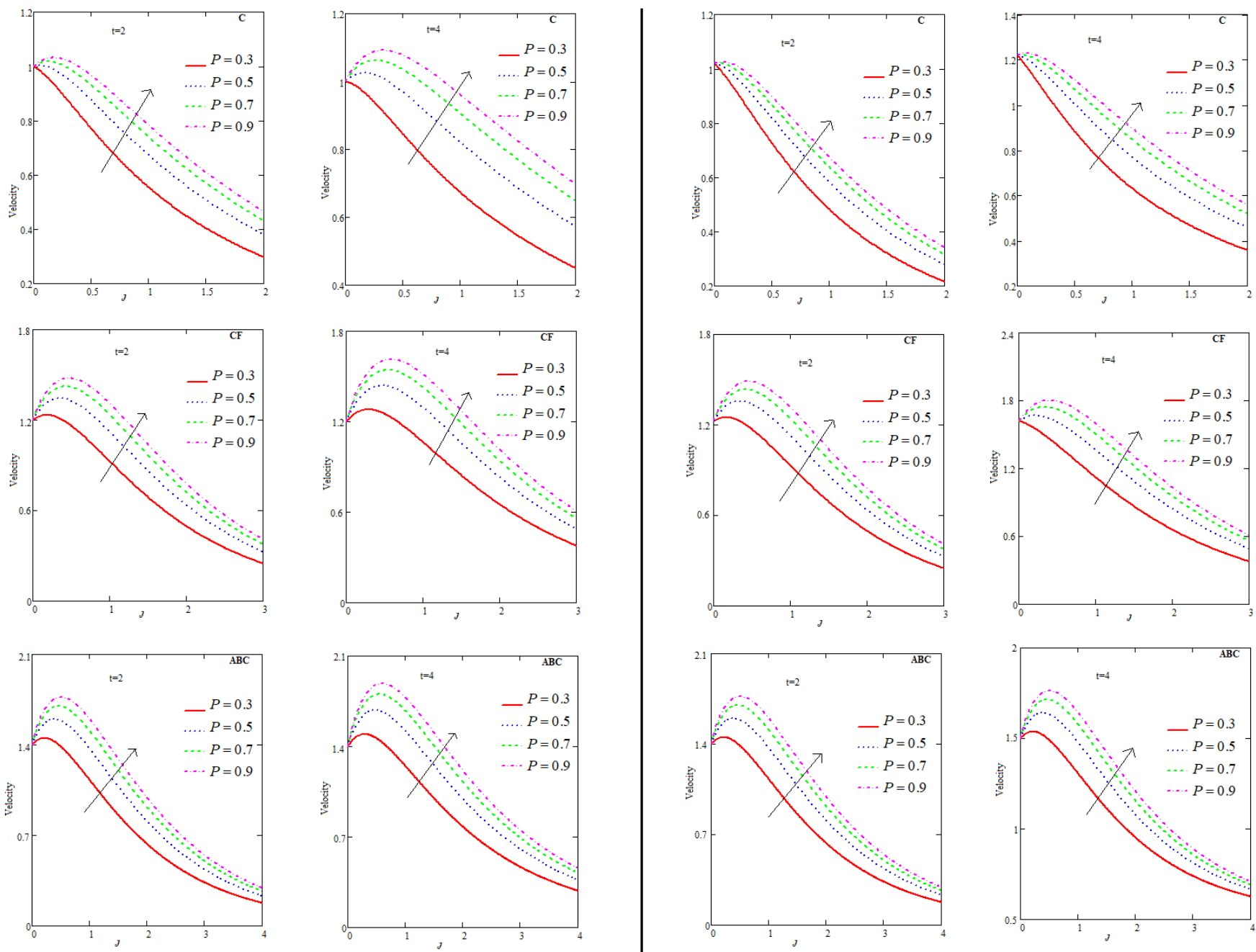

(a) Graphs for $d(t)=1$

(b) Graphs for $d(t)=e^{t}$

Figure 5. Graphs for two different cases of velocity with variable permittivity of medium and $M_{0}=2, S c=1.2, P r=1$, $G m=5, G r=5, A_{o}=0.5, \hbar_{2}=0.7, \aleph=0.2, \chi=0.1$ and $\kappa=0.9$.

\subsection{Effect of $\hbar_{2}$}

From Figure 6, it is obvious that CNT flow of a nano-fluid decreases with increase in $\hbar_{2}$. Moreover, unlike other fractional parameters, it shows a strange behavior, i.e., at certain point on $\jmath$-axis, $\hbar_{2}$ starts increasing and converges as $\jmath$ approaches infinity. It is minimum, moderate and maximum for $C, C F$ and $A B C$, respectively. 

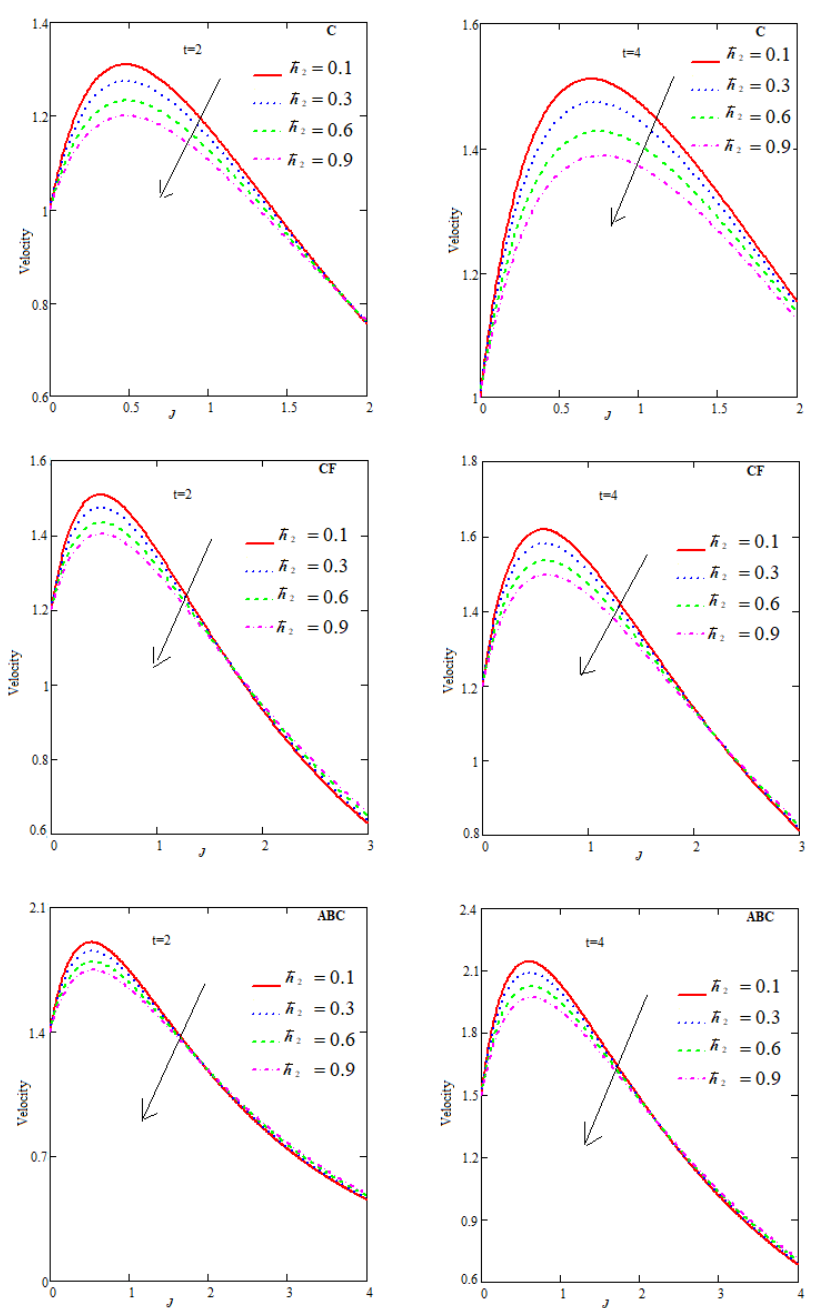

(a) Graphs for $d(t)=1$
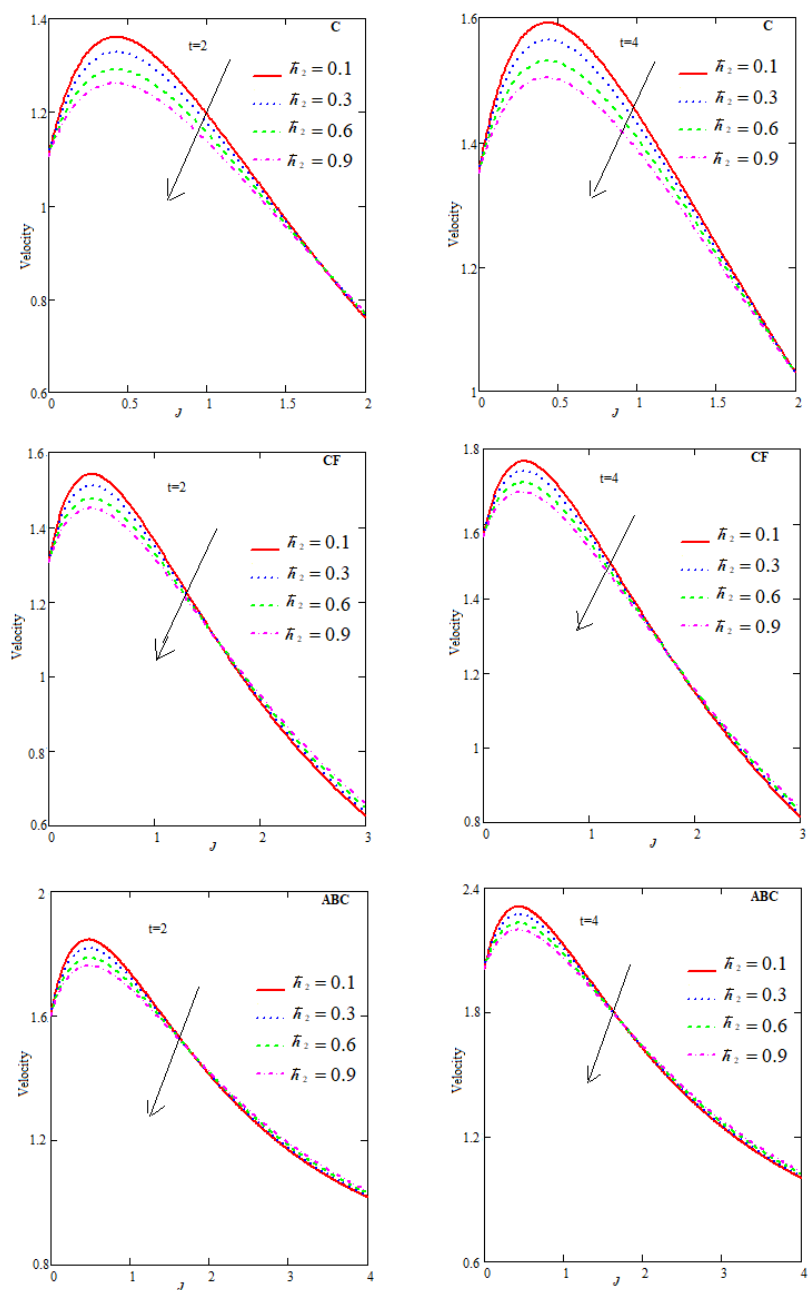

(b) Graphs for $d(t)=e^{t}$

Figure 6. Graphs for two different cases of velocity with variable 2 nd grade parameter and $M_{o}=1, S c=1.2, P r=1$, $\mathrm{Gm}=5, \mathrm{Gr}=5, A_{0}=0.5, P=2, \aleph=0.2, \chi=0.1$ and $\kappa=0.9$.

\subsection{Effect of $\kappa$}

The fluid flow increases the way $\kappa$ escalates as shown in Figure 7. It is greatest for $A B C$ fractional MHD model. The resultant velocity for $A B C$ model is good as compared to $C$ and $C F$. 

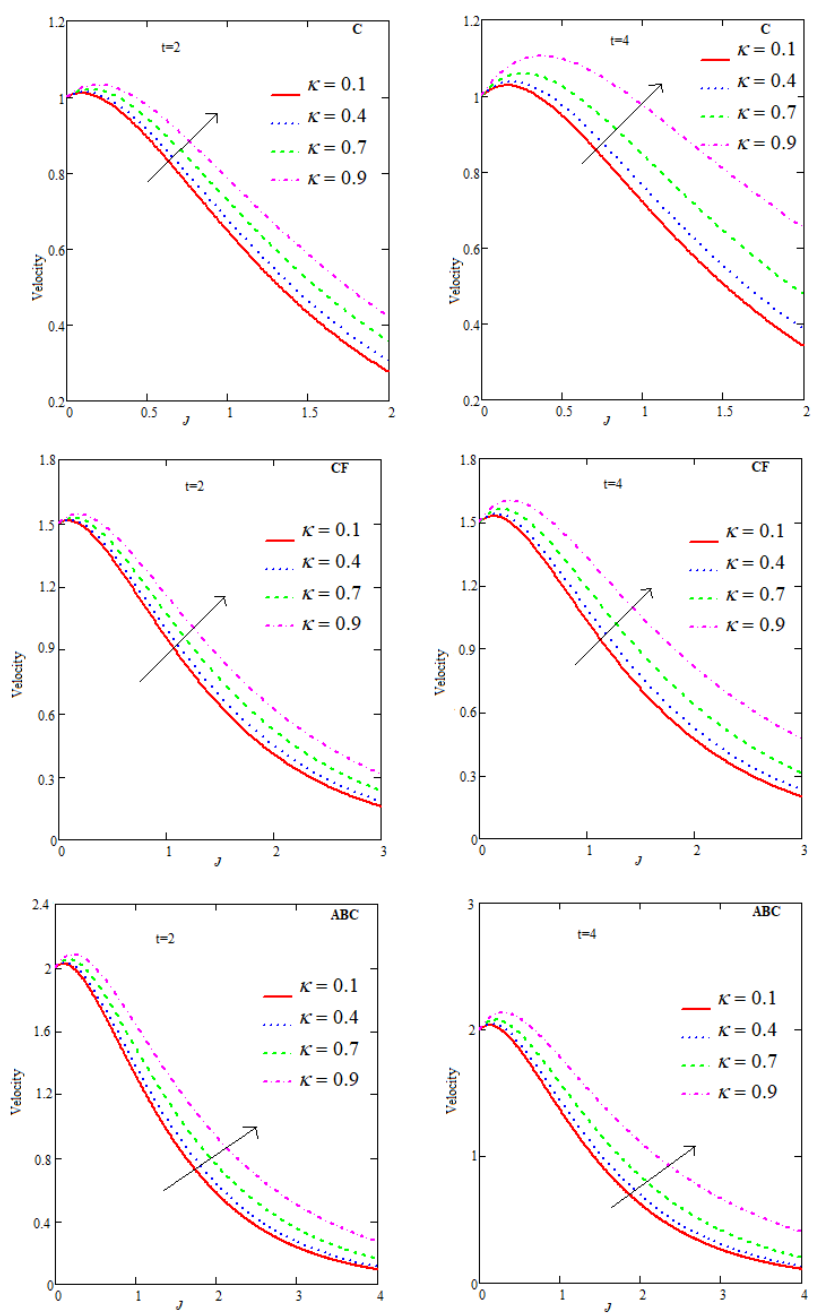

(a) Graphs for $d(t)=1$
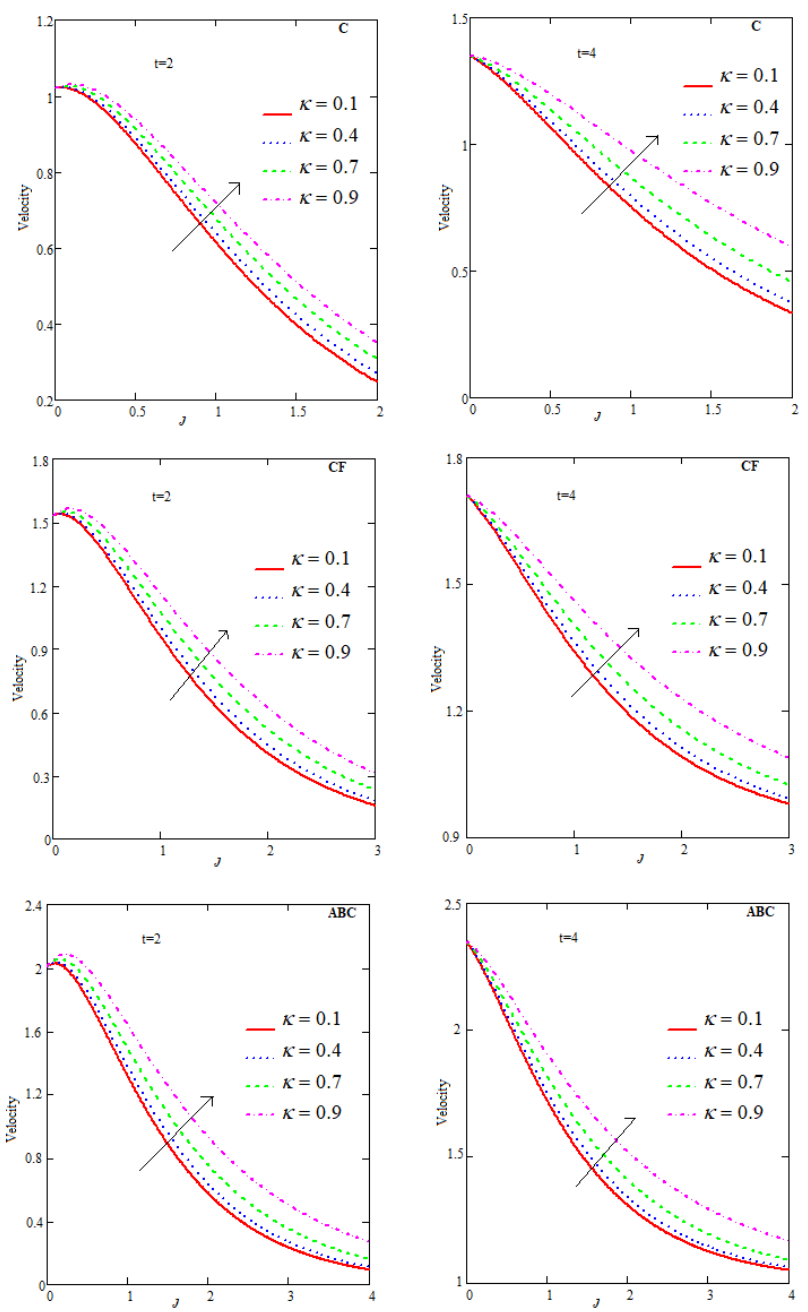

(b) Graphs for $d(t)=e^{t}$

Figure 7. Graphs for two different cases of velocity with variable chemical reaction parameter and $M_{o}=2, S c=1.2, P r=1$, $G m=5, G r=5, A_{o}=0.5, \hbar_{2}=0.7, \aleph=0.2, \chi=0.1$ and $P=0.3$.

\subsection{Effect of $M_{0}$}

Figure 8 illustrates that as magnetic field amplifies, fluid flow de-escalates. Lorentz force is a frictional force caused by magnetic field. When magnetic field maximizes, there is an increase in Lorentz force at outermost layer of fluid. this leads into decrease in velocity of nano-fluid. $A B C$ fractional model bears highest flow rate as compared to Caputo and Caputo-Fabrizio fractional MHD models. 

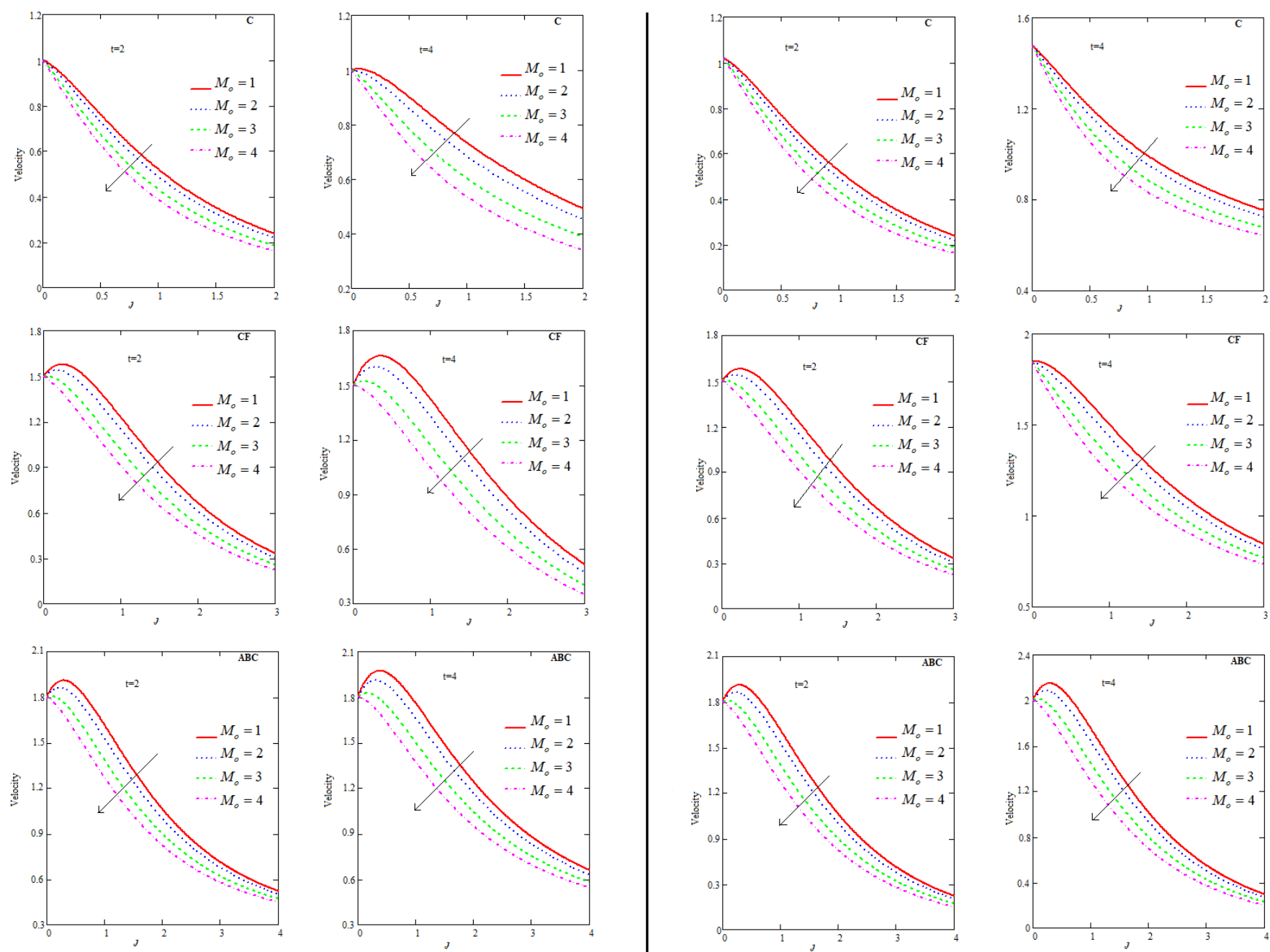

(a) Graphs for $d(t)=1$

(b) Graphs for $d(t)=e^{t}$

Figure 8. Graphs for two different cases of velocity with variable magnetic field and $P=0.3, S c=1.2, P r=1, G m=5$, $\mathrm{Gr}=5, A_{0}=0.5, \hbar_{2}=0.7, \aleph=0.2, \chi=0.1$ and $\kappa=0.9$.

\subsection{Effect of $\mathrm{Pr}$}

The flow profile declines, providing the values of $\mathrm{Pr}$ rise as displayed in Figure 9. $\mathrm{Pr}$ is directly and inversely proportional to momentum and thermal diffusivity, respectively. Fluid's viscosity increases, granting a decrease in thermal diffusivity. In response, the flow field decreases and it is minimal for Caputo. 

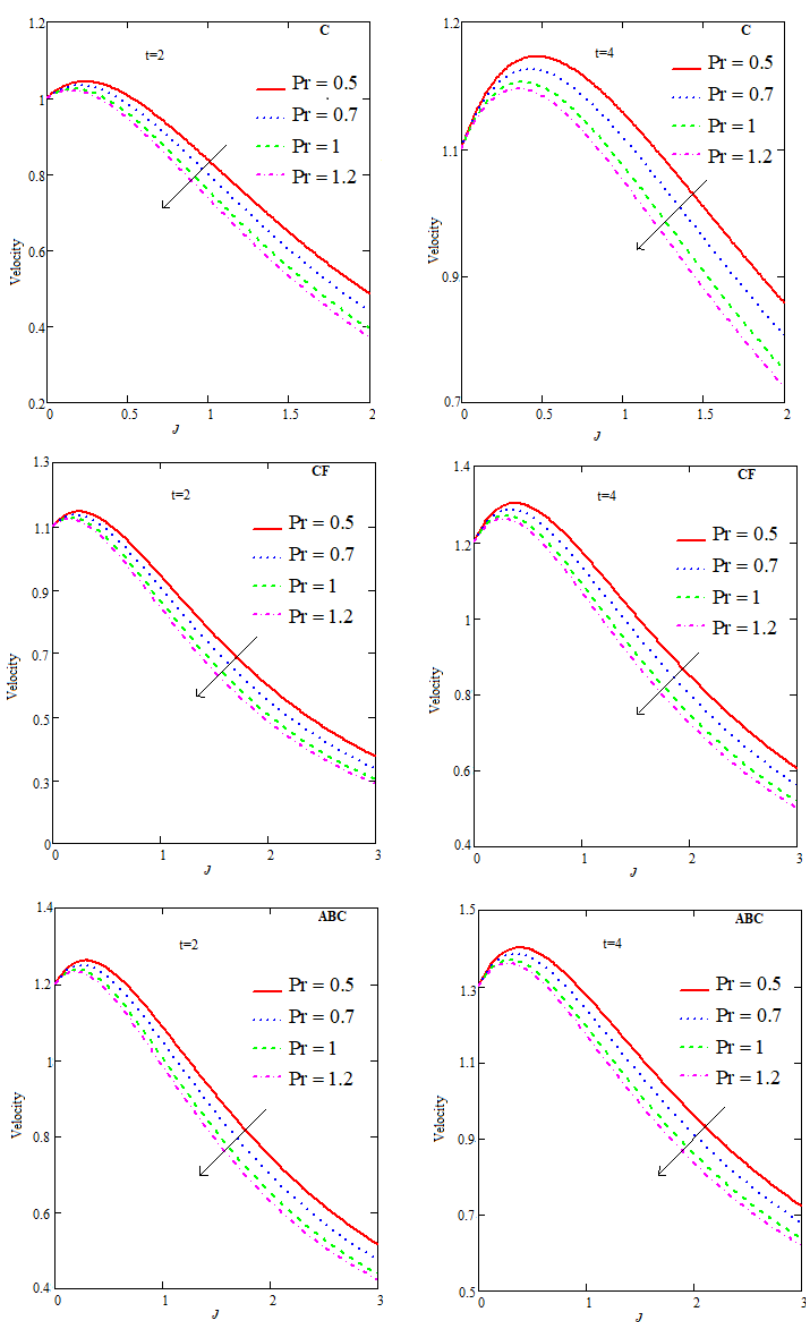

(a) Graphs for $d(t)=1$
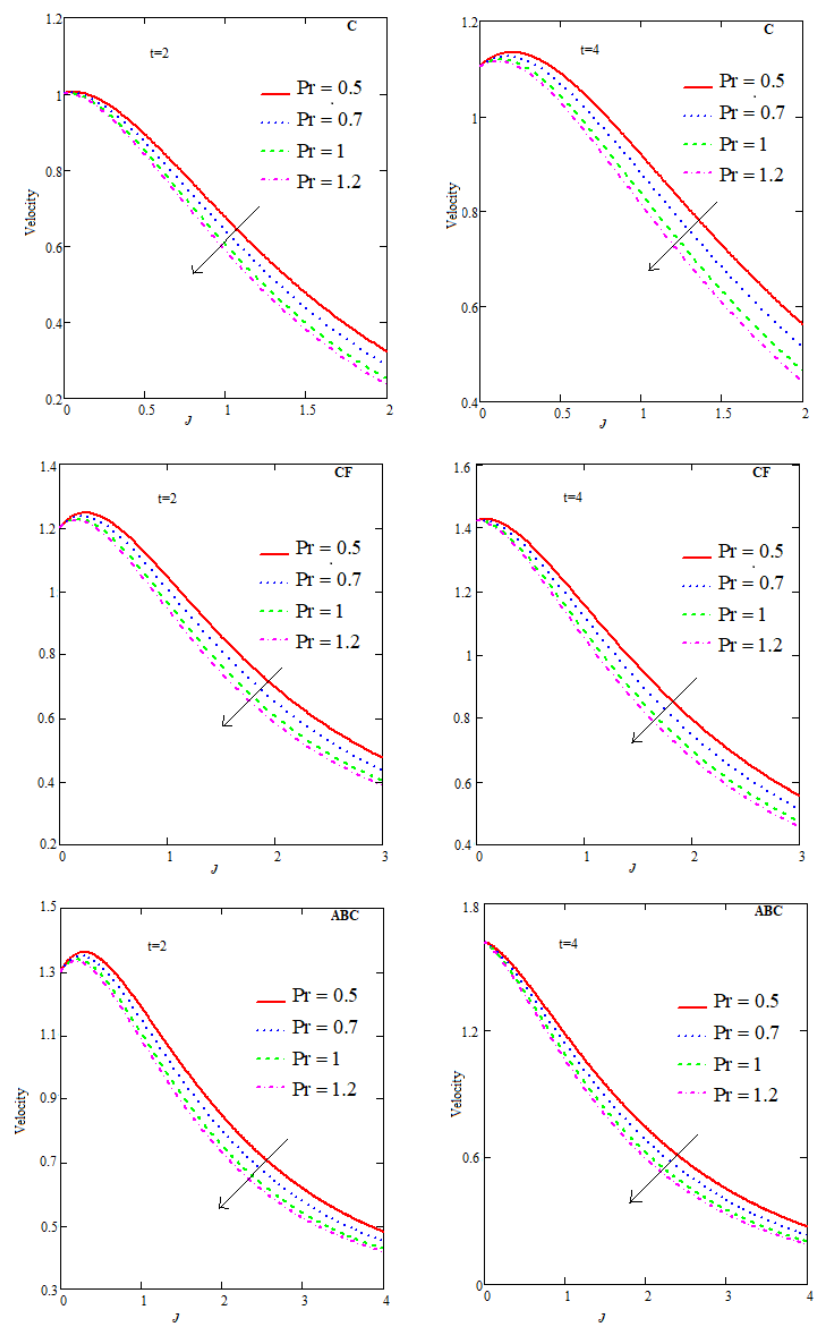

(b) Graphs for $d(t)=e^{t}$

Figure 9. Graphs for two different cases of velocity with variable Prandtl number and $M_{o}=2, S c=1.2, P=0.3, G m=5$, $\mathrm{Gr}=5, A_{0}=0.5, \hbar_{2}=0.7, \aleph=0.2, \chi=0.1$ and $\kappa=0.9$.

\subsection{Effect of $A_{o}$}

Figure 10 elaborates the effect of $A_{o}$ on velocity. Flow profile increases if there is an increment in heat generation. Velocity is maximal, average and minimal for $A B C, C F$ and $C$, respectively. 

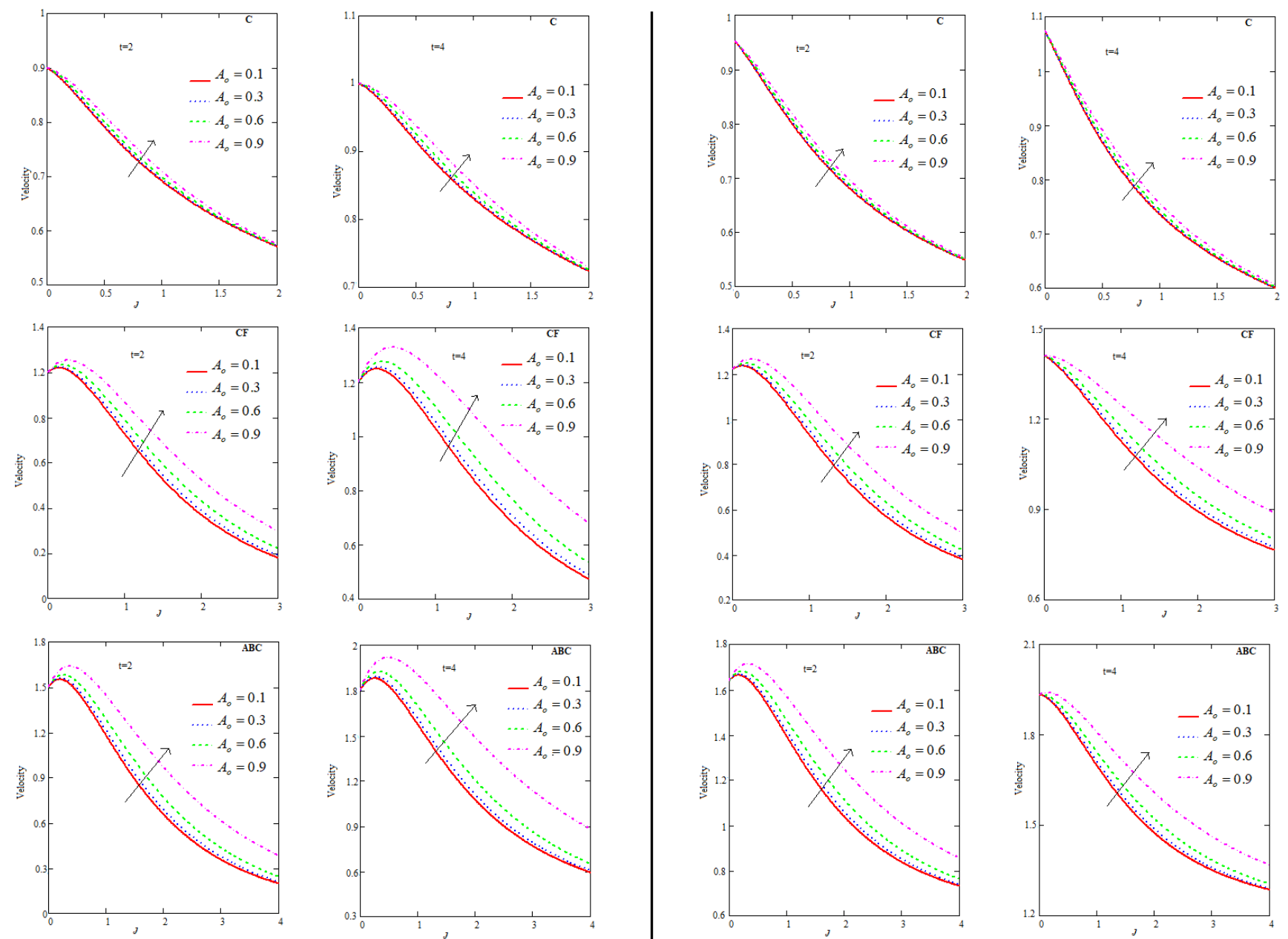

(a) Graphs for $d(t)=1$

(b) Graphs for $d(t)=e^{t}$

Figure 10. Graphs for two different cases of velocity with variable heat generation and $M_{o}=2, S c=1.2, \operatorname{Pr}=1, G m=5$, $\mathrm{Gr}=5, P=0.3, \hbar_{2}=0.7, \aleph=0.2, \chi=0.1$ and $\kappa=0.9$.

\subsection{Effect of}

In Figure 11, velocity field lessens when suction scales up. It is utmost for $A B C$ and lowest for $C$. While making comparison, velocity for the Atangana-Baleanu model is greatest because it has a non-local kernel. Velocity for $C F$ is greater than $C$. This is because $C F$ has a non-singular kernel that imitates $C$ with the singular kernel. 

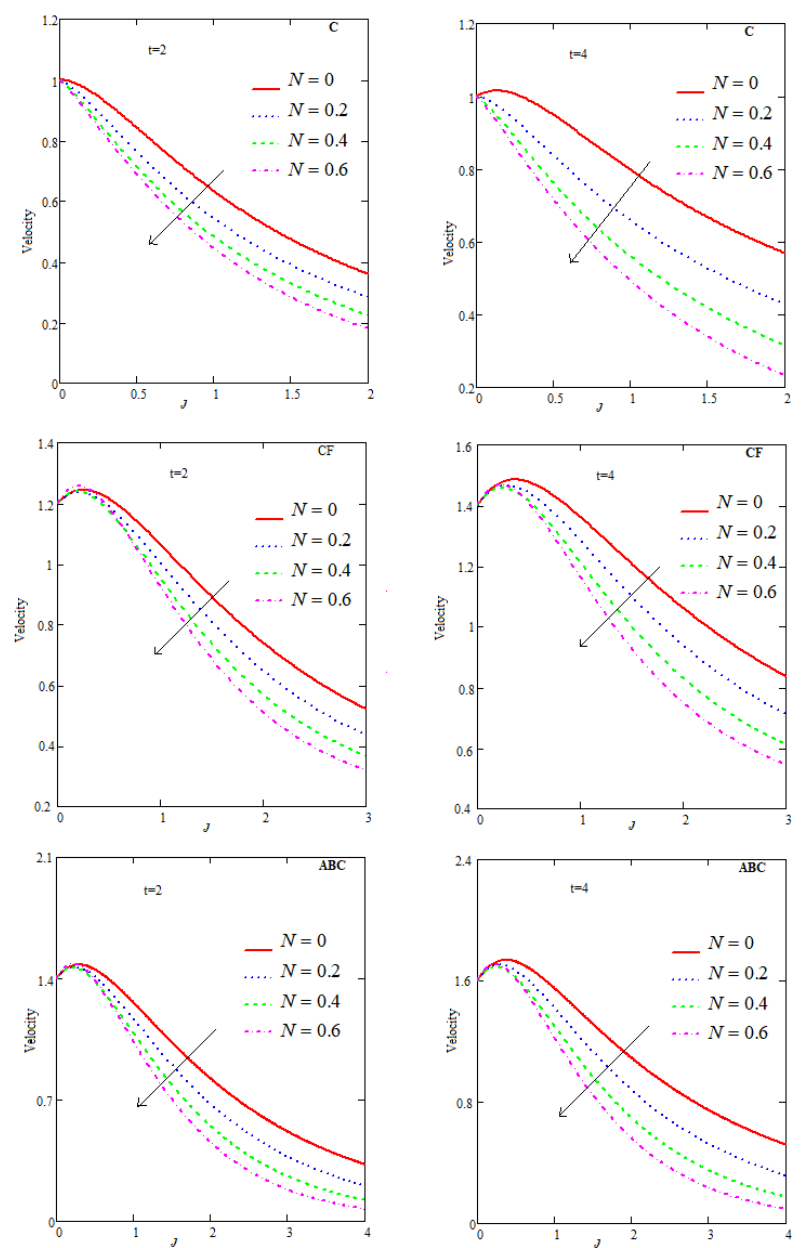

(a) Graphs for $d(t)=1$
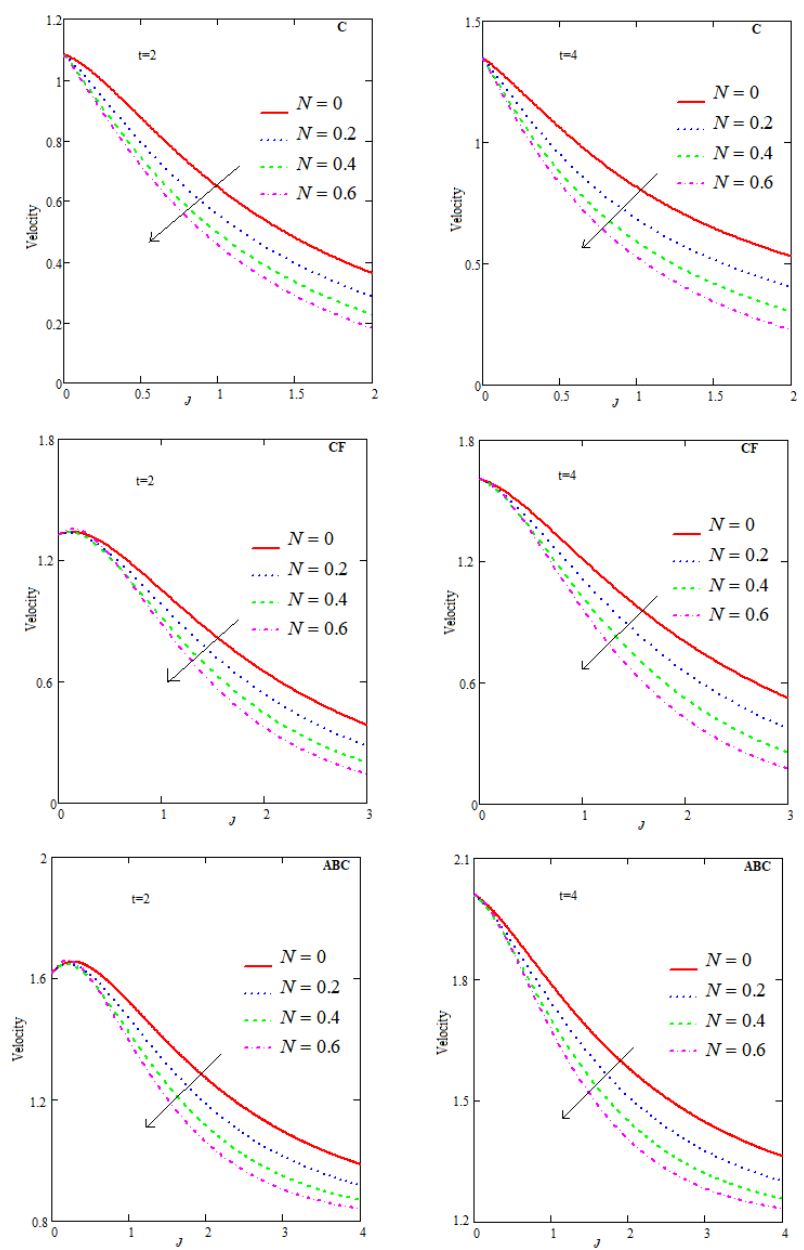

(b) Graphs for $d(t)=e^{t}$

Figure 11. Graphs for two different cases of velocity with variable Suction and $M_{o}=2, S c=1.2, \operatorname{Pr}=1, \mathrm{Gm}=5, \mathrm{Gr}=5$, $A_{o}=0.5, \hbar_{2}=0.7, P=0.3, \chi=0.1$ and $\kappa=0.9$.

\subsection{Effect of $S c$}

As the values of $S c$ increases, it increases kinematic viscosity, but decreases mass diffusivity. This successively descends flow profile of nano-fluid as presented in Figure 12. Velocity field for $A B C, C F$ and $C$ is supreme, adequate and least, respectively. 

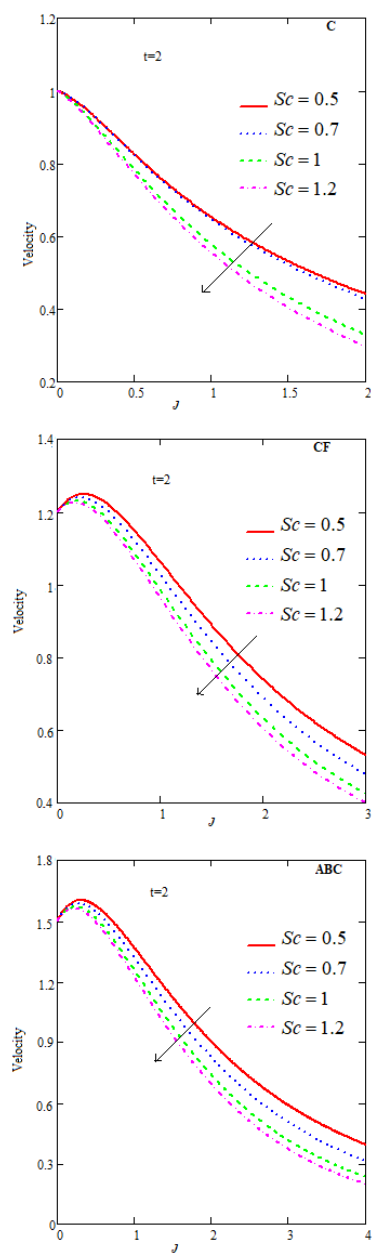

(a) Graphs for $d(t)=1$
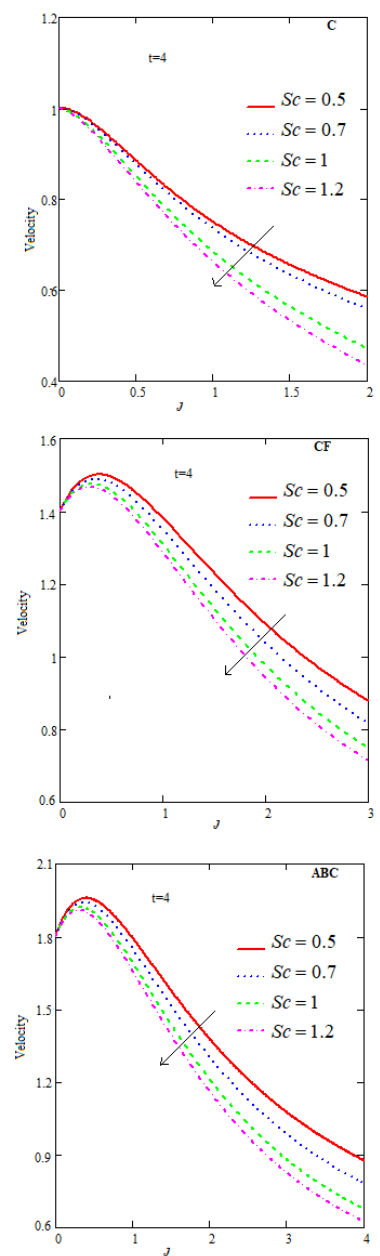
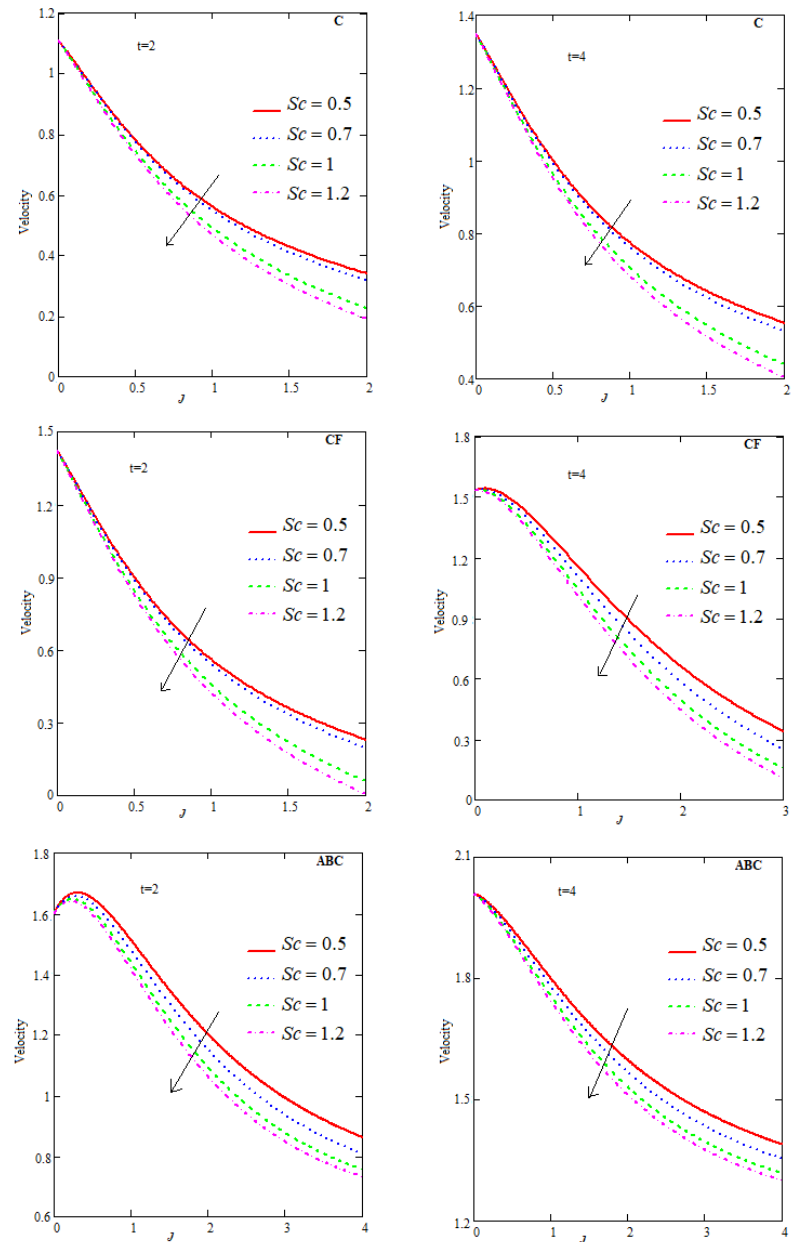

(b) Graphs for $d(t)=e^{t}$

Figure 12. Graphs for two different cases of velocity with variable Schmidt number and $M_{o}=2, P=0.3, P r=1, G m=5$, $\mathrm{Gr}=5, A_{0}=0.5, \hbar_{2}=0.7, \aleph=0.2, \chi=0.1$ and $\kappa=0.9$.

\section{Conclusions}

This article inquires time-dependent, MHD CNTs flow of nanofluid. The $C, C F$ and $A B C$ operators are used to construct equation for heat, mass and velocity. Solutions of model equations are presented by Laplace transform. Several graphs are given to illustrate impact of incipient parameters for solutions. Significant findings of this study are remarked as follows:

1. Fluid flow descends for $\chi, P, \hbar_{2}, P r, \aleph$ and $S c$.

2. Presence of resistive forces due to $M_{0}$, fluid flow decelerates.

3. Increment in flow field has been generated when $\mathrm{Gr}, \mathrm{Gm}, \kappa$ and $A_{0}$.

4. For $\aleph=0$, velocity of nano-fluid escalates. Whereas for $\aleph>0$, flow's velocity de-escalates.

5. Velocity of flow increases with increment in volumetric fraction for Caputo, CaputoFabrizio and Atangana-Baleanu models.

6. Velocity profile is maximum when $d(\tau)=e^{\tau}$ and minimum when $d(\tau)=1$.

7. Among $C, C F$ and $A B C$, flow profile is maximum for $A B C$.

Recently, there are some new techniques have been introduced in order to study fractional order models like modified generalized Taylor fractional series method (MGTFSM), fractional natural decomposition method (FNDM) and fractional Shehu transform, etc. In future, researchers can compare their results by the results we obtained in our research work using Caputo, Caputo-Fabrizio and Atangana-Baleanu fractional approach. 
Author Contributions: Conceptualization, N.I.; methodology, J.A.; software, A.A.; validation, M.B.R. and F.J.; formal analysis, A.A.; investigation, J.A.; data curation, F.J.; writing-original draft preparation, N.I. and J.A.; writing-review and editing, N.I. and F.J.; visualization, A.A.; supervision, A.A.; project administration, M.B.R.; Final checking, M.B.R. All authors have read and agreed to the published version of the manuscript.

Funding: This work has been supported by the Polish National Science Centre under the grant OPUS 18 No. 2019/35/B/ST8/00980.

Institutional Review Board Statement: Not applicable.

Informed Consent Statement: Not applicable.

Data Availability Statement: My Manuscript has no associated data.

Conflicts of Interest: The authors declare that they have no known competing financial interests or personal relationships that could have appeared to influence the work reported in this paper.

\section{Nomenclature}

\begin{tabular}{|c|c|}
\hline Symbol & Quantity \\
\hline$\kappa_{r}$ & Chemical reaction parameter \\
\hline$h$ & Coefficient of heat transfer $\left(\mathrm{Wm}^{-2} \mathrm{~K}^{-1}\right)$ \\
\hline$\widetilde{\Lambda}_{w}$ & Concentration level on the plate $\left(\mathrm{kgm}^{-3}\right)$ \\
\hline$\widetilde{\Lambda}_{\infty}$ & Concentration of the fluid far away from the plate $\left(\mathrm{kgm}^{-3}\right)$ \\
\hline$v_{0}$ & Constant suction velocity (s) \\
\hline$\rho_{n f}$ & Density of nano fluid $\left(\mathrm{kgm}^{-3}\right)$ \\
\hline$\mu_{n f}$ & Dynamic viscosity of nano fluid $\left(\mathrm{kgm}^{-1} \mathrm{~s}^{-1}\right)$ \\
\hline$\widetilde{\Lambda}$ & Fluid concentration $\left(\mathrm{kgm}^{-3}\right)$ \\
\hline$\widetilde{Y}$ & Fluid temperature $(\mathrm{K})$ \\
\hline$\widetilde{W}$ & Fluid velocity $\left(\mathrm{ms}^{-1}\right)$ \\
\hline$\chi$ & Fractional parameter \\
\hline$g$ & Gravitational acceleration $\left(\mathrm{ms}^{-2}\right)$ \\
\hline$A_{o}$ & Heat generation \\
\hline$v_{n f}$ & Kinematic viscosity of nano fluid $\left(\mathrm{m}^{2} \mathrm{~s}^{-1}\right)$ \\
\hline$u$ & Laplace transforms parameter \\
\hline$M_{o}$ & Magnetic field \\
\hline Gm & Mass Grashof number \\
\hline$\hbar_{1}$ & Material constant or 2 nd grade parameter \\
\hline$P_{1}$ & Permitivity of medium \\
\hline$\wp$ & Porosity \\
\hline $\operatorname{Pr}$ & Prandtl number \\
\hline Sc & Schmidt number \\
\hline Sh & Sherwood number \\
\hline$C_{p}$ & Specific heat at constant temperature $\left(\mathrm{jkg}^{-1} \mathrm{~K}^{-1}\right)$ \\
\hline$\aleph$ & Suction \\
\hline$\widetilde{\mathrm{Y}}_{w}$ & Temperature of fluid at the plate $(\mathrm{K})$ \\
\hline$\widetilde{Y}_{\infty}$ & Temperature of fluid far away from the plate $(\mathrm{K})$ \\
\hline$k$ & Thermal conductivity of the fluid $\left(\mathrm{Wm}^{-1} \mathrm{~K}^{-1}\right)$ \\
\hline Gr & Thermal Grashof number \\
\hline$\beta_{\widetilde{\Lambda}}$ & Volumetric coefficient of expansion for mass concentration $\left(\mathrm{m}^{3} \mathrm{~kg}^{-1}\right)$ \\
\hline$\beta_{\widetilde{Y}}$ & Volumetric coefficient of thermal expansion $\left(\mathrm{K}^{-1}\right)$ \\
\hline
\end{tabular}

\section{References}

1. Palani, G.; Abbas, I.A. Free convection MHD flow with thermal radiation from an impulsive started vertical plate. Non-Linear Anal. Model Control. 2009, 14, 73-84. [CrossRef]

2. Kumar, B.R.; Sivaraj, R. Heat and mass transfer in MHD viscoelastic fluid flow over a vertical cone and flat plate with variable viscosity. Int. J. Heat Mass Trans. 2013, 56, 370-379. [CrossRef]

3. Das, S.S.; Parija, S.; Padhy, R.K.; Sahu, M. Natural convection unsteady magneto-hydrodynamic mass transfer flow past an infinite vertical porous plate in presence of suction and heat sink. Int. J. Energy Environ. 2012, 3, $209-222$. 
4. Atangana, A. On the new fractional derivative and application to nonlinear Fishers reaction-diffusion equation. Appl. Math. Comput. 2016, 273, 948-956.

5. Tarasova, V.V.; Tarasov, V.E. Economic interpretation of fractional derivatives. Prog. Fract. Diff. Appl. 2017, 3, 1-7. [CrossRef]

6. Yang, X.J.; Machado, J.A.T. A new fractional operator of variable order: Application in the description of anomalous diffusion. Phys. A 2017, 481, 276-283. [CrossRef]

7. Zhuravkov, M.A.; Romanova, N.S. Review of methods and approaches for mechanical problem solutions based on fractional calculus. Math. Mech. Solids. 2016, 21, 595-620 [CrossRef]

8. Cao, Z.; Zhao, J.; Wang, Z.; Liu, F.; Zheng, L. MHD flow and heat transfer of fractional Maxwell viscoelastic nanofluid over a moving plate. J. Mol. Liq. 2016, 222, 1121-1127. [CrossRef]

9. Pandey; Kumar, A.; Kumar, M. Effect of viscous dissipation and suction/injection on MHD nanofluid flow over a wedge with porous medium and slip. Alex. Eng. J. 2016, 55, 3115-3123. [CrossRef]

10. Murshed, S.M.S.; Castro, C.A.N.D.; Lourenço, M.J.V.; Lopes, M.L.M.; Santos, F.J.V. A review of boiling and convective heat transfer with nanofluids. Renew. Sustain. Energy Rev. 2011, 15, 2342-2354. [CrossRef]

11. Abro, K.A.; Khan, I.; Tassaddiq, A. Application of Atangana-Baleanu fractional derivative to convection flow of MHD Maxwell fluid in a porous medium over a vertical plate. Math. Model. Nat. Phen. 2018, 13, 1. [CrossRef]

12. Saqib, M.; Kasim, A.R.M.; Mohammad, N.F.; Ching, D.L.C.; Shafie, S. Application of fractional derivative without singular and local kernel to enhanced heat transfer in CNTs nanofluid over an inclined plate. Symmetry 2020, 12, 768. [CrossRef]

13. Wong, K.V.; Leon, O.D. Applications of nanofluids: Current and future. Nanotechnol. Energy 2017, $105-132$.

14. Lin, Y.H.; Kang, S.W.; Chen, H.L. Effect of silver nanofluid on pulsating heat pipe thermal performance. Appl. Therm. Eng. 2008, 28, 1312-1317. [CrossRef]

15. Saqib, M.; Khan, I.; Shafie, S. Application of Atangana-Baleanu fractional derivative to MHD channel flow of CMC-based-CNT's nanofluid through a porous medium. Chaos Solitons Fractals 2018, 116, 79-85. [CrossRef]

16. Ikram, M.D.; Asjad, M.I.; Akgül, A.; Baleanu, D. Effects of hybrid nanofluid on novel fractional model of heat transfer flow between two parallel plates. Alex. Eng. J. 2021, 60, 3593-3604. [CrossRef]

17. Maiti, S.; Shaw, S.; Shit, G.C. Fractional order model for thermochemical flow of blood with Dufour and Soret effects under magnetic and vibration environment. Colloids Surf. B Biointerfaces 2021, 197, 111395. [CrossRef]

18. Sreedevi, P.; Reddy, P.S.; Rao, K.V.S.N.; Chamkhab, A.J. Heat and mass transfer flow over a vertical cone through nanofluid saturated porous medium under convective boundary condition suction/injection. J. Nanofluids 2017, 6, 478-486. [CrossRef]

19. Du, M.; Wang, Z.; Hu, H. Measuring memory with the order of fractional derivative. Sci. Rep. 2013, 3, 3431. [CrossRef] [PubMed]

20. Hayat, T.; Muhammad, T.; Shehzad, S.A.; Alsaedi, A. An analytical solution for magnetohydrodynamic Oldroyd-B nanofluid flow induced by a stretching sheet with heat generation/absorption. Int. J. Therm. Sci. 2017, 111, 274-288. [CrossRef]

21. Ghalandari, M.; Maleki, A.; Haghighi, A.; Shadloo, M.S.; Nazari, M.A.; Tlili, I. Applications of nanofluids containing carbon nanotubes in solar energy systems: A review. J. Mol. Liq. 2020, 313, 113476. [CrossRef]

22. Rao, Y. Nanofluids: Stability, phase diagram, rheology and applications. Particuology 2010, 8, 549-555. [CrossRef]

23. Lim, A.E.; Lim, C.Y.; Lam, Y.C.; Taboryski, R. Electroosmotic flow in microchannel with black silicon nanostructures. Micromachines 2018, 9, 229. [CrossRef]

24. Lim, A.E.; Lim, C.Y.; Lam, Y.C.; Taboryski, R.; Wang, S.R. Effect of nanostructures orientation on electroosmotic flow in a microfluidic channel. Nanotechnology 2017, 28, 255-303. [CrossRef]

25. Alawi, O.A.; Mallah, A.R.; Kazi, S.N.; Sidik, N.A.; Najafi, G. Thermophysical properties and stability of carbon nanostructures and metallic oxides nanofluids. J. Therm. Anal. Calorim. 2019, 135, 1545-1562. [CrossRef]

26. Pandikunta, S.; Tamalapakula, P.; Reddy, N.B. Inclined Lorentzian force effect on tangent hyperbolic radiative slip flow imbedded carbon nanotubes: Lie group analysis. J. Comput. Appl. Res. Mech. Eng. 2020, 10, 85-99.

27. Aghamajidi, M.; Yazdi, M.; Dinarvand, S.; Pop, I. Tiwari-Das nanofluid model for magnetohydrodynamics (MHD) naturalconvective flow of a nanofluid adjacent to a spinning down-pointing vertical cone. Propuls. Power Res. 2018, 7, 78-90. [CrossRef]

28. Reddy, S.R.; Reddy, P.B.; Chamkha, A.J. Heat Transfer Analysis Of MHD CNTS Nanofluids Flow Over a Stretching Sheet. Spec. Top. Rev. Porous Media Int. J. 2020, 11, 133-147. [CrossRef]

29. Upreti, H.; Rawat, S.K.; Kumar, M. Radiation and non-uniform heat sink/source effects on 2D MHD flow of CNTs-H2O nanofluid over a flat porous plate. Multidiscip. Model. Mater. Struct. 2019, 16, 791-809. [CrossRef]

30. Upreti, H.; Pandey, A.K.; Kumar, M.; Makinde, O.D. Ohmic heating and non-uniform heat source/sink roles on 3D Darcy-Forchheimer flow of CNTs nanofluids over a stretching surface. Arab. J. Sci. Eng. 2020, 45, 7705-7717. [CrossRef]

31. Hussain, Z.; Hayat, T.; Alsaedi, A.; Ahmad, B. Three-dimensional convective flow of CNTs nanofluids with heat generation/absorption effect: a numerical study. Comput. Methods App. Mech. Eng. 2018, 329, 40-54. [CrossRef]

32. Alsagri, A.S.; Nasir, S.; Gul, T.; Islam, S.; Nisar, K.S.; Shah, Z.; Khan, I. MHD thin film flow and thermal analysis of blood with CNTs nanofluid. Coatings 2019, 9, 175. [CrossRef]

33. Kumam, P.; Tassaddiq, A.; Watthayu, W.; Shah, Z.; Anwar, T. Modeling and simulation based investigation of unsteady MHD radiative flow of rate type fluid; a comparative fractional analysis. Math. Comput. Simul. 2021, in press.

34. Saqib, M.; Khan, I.; Shafie, S.; Mohamad, A.Q. Shape effect on MHD flow of time fractional Ferro-Brinkman type nanofluid with ramped heating. Sci. Rep. 2021, 11, 3725. [CrossRef] 
35. Roohi, R.; Heydari, M.H.; Bavi, O.; Emdad, H. Chebyshev polynomials for generalized Couette flow of fractional Jeffrey nanofluid subjected to several thermochemical effects. Eng. Comput. 2021, 37, 579-595. [CrossRef]

36. Maiti, S.; Shaw, S.; Shit, G.C. Fractional order model of thermo-solutal and magnetic nanoparticles transport for drug delivery applications. Colloids Surf. B Biointerfaces 2021, 203, 111754. [CrossRef]

37. Abro, K.A. Role of fractal-fractional derivative on ferromagnetic fluid via fractal Laplace transform: A first problem via fractal-fractional differential operator. Eur. J. Mech-B/Fluids. 2021, 85, 76-81. [CrossRef]

38. Ahmad, I.; Nazar, M.; Ahmad, M.; Nisa, Z.U.; Shah, N.A. MHD-free convection flow of CNTs differential type nanofluid over an infinite vertical plate with first-order chemical reaction, porous medium, and suction/injection. Math. Methods Appl. Sci. 2020. [CrossRef]

39. Imran, M.A.; Khan, I.; Ahmad, M.; Shah, N.A.; Nazar, M. Heat and mass transport of differential type fluid with non-integer order time-fractional Caputo derivatives. J. Mol. Liq. 2017, 229, 67-75. [CrossRef]

40. Al-Refai, M.; Abdeljawad, T. Analysis of the fractional diffusion equations with fractional derivative of non-singular kernel. Adv. Diff. Eq. 2017, 2017, 315. [CrossRef]

41. Abdeljawad, T.; Baleanu, D. On fractional derivatives with generalized Mittag-Leffler kernels. Adv. Diff. Eq. 2018, 2018, 468. [CrossRef]

42. Jarad, F.; Abdeljawad, T.; Hammouch Z. On a class of ordinary differential equations in the frame of Atangana-Baleanu fractional derivative. Chaos Solitons Fractals 2018, 117, 16-20. [CrossRef]

43. Stehfest, H.A. Numerical inversion of Laplace transforms. Commun. ACM 1970, 13, 9-47. 